\title{
Bounded interpersonal inferences and decision making ${ }^{\star}$
}

\author{
Mamoru Kaneko ${ }^{1}$ and Nobu-Yuki Suzuki ${ }^{2}$ \\ 1 Institute of Policy and Planning Sciences, University of Tsukuba, Ibaraki 305-8573, JAPAN \\ (e-mail: kaneko@shako.sk.tsukuba.ac.jp) \\ 2 Department of Mathematics, Faculty of Science, Shizuoka University, Ohya, \\ Shizuoka 422-8529, JAPAN (e-mail: smnsuzu@ipc.shizuoka.ac.jp)
}

Received: August 31, 2000; revised version: April 9, 2001

\begin{abstract}
Summary. Individual decision making is based on predictions about other players' choices as well as on valuations of reactions to predictions. In this sense, a player has a prediction-decision criterion for decision making. We develop a theory of prediction-decision criteria, which enables us to capture new phenomena on individual decision making in games. The decision making situation is described in the epistemic logic GL $_{E F}$ of shallow depths. There, each player considers his and other players' decision making down to some shallow depths. It is a point of our theory to investigate inferential complexities of interpersonal introspections. In particular, we can discuss a minimal epistemic inferential structure for prediction-decision making. We will find parallel structures in decision making and prediction making, which is called an inner parallelism. The climax of the paper is the consideration of inner parallelisms of prediction-decision making.
\end{abstract}

Keywords and Phrases: Shallow epistemic depth, Prediction-decision criterion, Epistemic structure, Complexity of interpersonal introspection, Inner parallelism.

JEL Classification Numbers: C60, C69, D89.

\section{Introduction}

It is a central theme of game theory to investigate how people behave in interactive situations. A solution concept describes decision (behavior) criteria of

\footnotetext{
* The authors are partially supported by Grant-in-Aids for Scientific Research No. 13430001 and No. 13640111, Japan Society for the Promotion of Science. The second author is also supported by the Sumitomo Foundation. The authors thank Oliver Schulte and Jeffrey J. Kline for detailed comments on earlier versions of this paper.
} 
people and the resulting outcomes from these criteria. Such an investigation is called a solution theory, and various theories have been considered in the literature of game theory. Each solution theory may involve two types of interpersonal considerations: (a) interpersonal introspections in the mind of a player; and (b) interpersonal interactions at physical levels. Exchanges of messages in an extensive game are examples for (b). In this paper, we restrict our attention to (a). That is, we consider structures of interpersonal introspections required for decision making in game situations.

A typical characteristic of extant solution theories is the pursuit of "rationality" in resulting outcomes. This imposes payoff maximization for a decision maker and symmetrically for the other players even in the mind of the decision maker. We take the different view that decision criteria are more arbitrary than ones typically considered in game theory. Such an arbitrariness is due the fact that other players' minds are largely hypothetical. Under this view, we investigate structures of interpersonal introspections required for individual decision making, putting emphasis on the shallowness of interpersonal introspections.

The different view we adopt liberates us from the pursuit of "rationality" in decision making and its resulting outcomes. Rather than talking about "rationality" in outcomes, we would like to retain the term "rationality" to describe an attribute of the reasoning ability of a player. We call this the logical rationality of a player.

Extant solution theories typically involve some or many transcendental factors. This is caused by the fact that a simple decision criterion often fails to recommend a decision. The avoidance of such a failure leads to the pursuit of "rationality". Here, we treat rather simple and naive decision criteria. First, we exclude mixed strategies from our consideration, and a fortiori, we do not consider solution concepts related to the literature of "perfection". Even for epistemic requirements, we also avoid assumptions containing transcendentalities such as common knowledge. Thus, we find only a few simple and naive decision criteria in the literature of game theory, but can find a lot from our real life. In this paper, we investigate the structure of such simple and naive criterion from the viewpoint of the logical rationality of players.

Since decision making may involve predictions about what other players would choose, decision criteria are more accurately described as predictiondecision criteria. Different prediction-decision criteria may require different interpersonal introspections. For example, the dominant strategy criterion requires a player to think only about his own payoff function, and requires no interpersonal introspections. The same is true for the maximin decision criterion. Another example is that a player assumes, in his prediction, the dominant strategy criterion for the other player and chooses a best strategy (response) to the predicted strategy. In this example, truly interpersonal introspections are involved.

To facilitate considerations of interpersonal introspections for decision making, we adopt the epistemic logics of shallow depths developed in Kaneko and Suzuki [7], [8] and [9]. This logical system is denoted by GL $E F$. The subscripts $E$ and $F$ of $\mathrm{GL}_{E F}$ are called, respectively, 


\section{(1): descriptive epistemic structure;}

\section{(2): inferential epistemic structure.}

Both impose bounds on epistemic depths, where epistemic depths are the nested structure of beliefs of players having the form: player $i_{1}$ believes that $i_{2}$ believes $\ldots i_{m}$ believes something. The former, $E$, is the bounds of epistemic depths for a statement, and the latter, $F$, those of interpersonal introspections to infer the statement. It may be the case that a player has beliefs about other players but may make a decision without using his beliefs on other players. In this case, the notion of (1) is complicated, but that of (2) is simple. Thus, we need to distinguish between (1) and (2). A minimal inferential epistemic structure for a given statement is a key for this distinction.

To differentiate the above two notions more clearly, consider another example: pure default decision criterion. Suppose that player $i$ gives up thinking about the game and adopts his first strategy as his default decision. Then he needs no logical reasoning for the choice of the first (default) strategy. On the other hand, player $i$ still needs to be conscious of what his default decision is. This consciousness requires $E$ to be larger than $F$. In this case, the minimal inferential epistemic structure $F$ is null, but $E$ contains at least depth 1 . This will be discussed in Section 7.

We analyze the structure of prediction-decision making, while simultaneously developing a theory of epistemic logics with shallow depths for our analysis. Therefore, we call our theoretical development the logico-game analysis to differentiate our development from other extant theories.

Since the logico-game analysis in this paper will have a long development of both game theoretic problems and epistemic logic $\mathrm{GL}_{E F}$, it would be helpful to state that the climax of the development is the consideration of inner parallelisms of prediction-decision making in Section 8. Inner parallelisms mean that a parallel form of prediction-decision making is found in each player's prediction making. This is explicitly argued and shown, using certain meta-theorems obtained for $\mathrm{GL}_{E F}$. This result relies upon our basic assumptions that the same logical rationality is given to each player, the investigator (observer) and players imagined in the mind of each player.

Let us explain our undertaking from a different point of view. In Kaneko [4], epistemic logic KD4 ${ }^{n}$ with the belief operators $\mathrm{B}_{1}, \ldots, \mathrm{B}_{n}$ of players $1, \ldots, n$ is discussed as taking the central position. In $\mathrm{KD} 4^{n}$, formulae having nested occurrences of $\mathrm{B}_{1}, \ldots, \mathrm{B}_{n}$ in any depths are allowed, and the Necessitation Rule may be applied arbitrarily many times in proofs. On the other hand, human interpersonal epistemic introspections often stop at very shallow levels. The purpose of introducing $\mathrm{GL}_{E F}$ is to take this limitation of human reasoning seriously. This is directly related to the above game theoretical motivation in that we avoid transcendentalities and treat prediction-decision criteria having only shallow interpersonal introspections.

The logical system $\mathrm{GL}_{E F}$ is obtained by imposing two types of restrictions on $\mathrm{KD}^{n}$, rather than $\mathrm{KD}^{n}$, by means of descriptive $E$ and inferential $F$. Nested 
occurrences of belief operators in formulae and proofs are restricted by $E$ and $F$, respectively. We emphasize that $F$ is a subset of $E$ and may be smaller than $E$. Thus, only shallower interpersonal introspections are required for the logical rationality for decision making than for the description of the epistemic situation.

We close this introduction with two remarks. The first is on our choice of a presentation style of $\mathrm{GL}_{E F}$. In Kaneko [4], the Hilbert-style formal system and Kripke-style semantics for $\mathrm{KD}^{n}$ are primarily discussed. We may adopt these types for $\mathrm{GL}_{E F}$, but for our considerations of game theoretical problems as well as some logical problems, either is inconvenient in the sense that we need to prepare a lot of lemmas. Instead, we present $\mathrm{GL}_{E F}$ in the Gentzen-style sequent calculus, which enables us to go directly to our problems. The Kripke-style semantics for $\mathrm{GL}_{E F}$ is found in Kaneko and Suzuki [9].

The second remark is on the exclusion of Axiom 4 (Positive Introspection Axiom) from $\mathrm{GL}_{E F}$. One reason for this exclusion is that our focus is the consideration of interpersonal introspections but not intrapersonal ones. Another reason is that the exclusion makes our meta-theoretical treatments much easier. Nevertheless, the results given in this paper essentially remain to hold, which will be discussed in the Appendix (Section 11).

The paper is organized as follows: In Section 2, we prepare basic game theoretic notions and various prediction-decision criteria in the nonformalized language. In Section 3, we give the definitions of formulae and epistemic structures. In Section 4, we give $\mathrm{GL}_{E F}$ and state the cut-elimination theorem for it, and we illustrate some provable statements on prediction-decision making. One important result, called the Decomposition Theorem, is given there. This states that the prediction-decision statements for $n$ players are decomposed into $n$ independent statements of individual prediction-decision making. This does not depend upon structures of prediction-decision criteria.

In Section 5, we give the general definition of a prediction-decision criterion, and see that various examples are special cases of this general definition. Section 6 presents various meta-theorems to be used for evaluations of predictiondecision making in a game. In Section 7, we consider minimal inferential epistemic structures for prediction-decision making with various criteria. Section 8 is the climax of this paper, in which we discuss inner parallelisms in predictiondecision making. In Section 9 suggests a further development such as compound prediction-decision criteria. Section 10 gives concluding remarks. Section 11 gives an appendix on the treatment of the Axiom 4 (Positive Introspection). We append the list of symbols for the reader's use.

\section{Some game theoretic notions}

In this section, we review basic game theoretical notions, and also give various prediction-decision criteria. Some are standard in the game theoretical literature, and others are found in our ordinary life. Such a variety of prediction-decision criteria are important to understand the scope of our logico-game analysis. 


\subsection{Basic notions and simple examples}

Consider an $n$-person finite noncooperative game $g=\left(g_{1}, \ldots, g_{n}\right)$ in strategic form. The set of players is denoted by $N:=\{1, \ldots, n\}$. Each player $i \in N$ has $\ell_{i}$ pure strategies $\left(\ell_{i} \geq 2\right)$. We assume throughout the paper that the players do not play mixed strategies. The set of player $i$ 's (pure) strategies is denoted by $S_{i}:=\left\{\mathbf{s}_{i 1}, \ldots, \mathbf{s}_{i \ell_{i}}\right\}$ for $i \in N$. His payoff function is a real-valued function $g_{i}$ on $S:=S_{1} \times \cdots \times S_{n}$. An element $s=\left(s_{1}, \ldots, s_{n}\right) \in S$ is called a strategy profile. For $s=\left(s_{1}, \ldots, s_{n}\right) \in S$, let $s_{-i}=\left(s_{1}, \ldots, s_{i-1}, s_{i+1}, \ldots, s_{n}\right)$. This is an element of $S_{-i}:=S_{1} \times \cdots \times S_{i-1} \times S_{i+1} \times \cdots \times S_{n}$. We write often $s=\left(s_{1}, \ldots, s_{n}\right) \in S$ as $s_{i} ; s_{-i}$.

A strategy $s_{i} \in S_{i}$ is a best strategy (response) to $s_{-i}$ iff $g_{i}\left(s_{i} ; s_{-i}\right) \geq$ $g_{i}\left(t_{i} ; s_{-i}\right)$ for all $t_{i} \in S_{i}$. We say that $s_{i}$ is a dominant strategy iff $s_{i}$ is a best strategy to $s_{-i}$ for any $s_{-i} \in S_{-i}$. A dominant strategy satisfies payoff maximization whatever the other players choose. We also consider the concept of an undominated strategy to discuss prediction-making. We say that $t_{i}$ dominates $s_{i}$ iff $g_{i}\left(t_{i} ; s_{-i}\right) \geq g_{i}\left(s_{i} ; s_{-i}\right)$ for all $s_{-i} \in S_{-i}$ and $g_{i}\left(t_{i} ; s_{-i}\right)>g_{i}\left(s_{i} ; s_{-i}\right)$ for some $s_{-i} \in S_{-i}$. A strategy $s_{i}$ is an undominated strategy iff no $t_{i} \in S_{i}$ dominates $s_{i}$. Note that an undominated strategy may not satisfy payoff maximization.

In the game $g^{1}=\left(g_{1}^{1}, g_{2}^{1}\right)$ of Table 1 (Prisoner's Dilemma), the second strategy $\mathbf{s}_{i 2}$ for each player $i$ is a dominant strategy and is undominated. The game $g^{2}=\left(g_{1}^{2}, g_{2}^{2}\right)$ of Table 2 is obtained from game $g^{1}$ only by changing payoff 6 in the north-east corner to 2 . In game $g^{2}$, player 1 has the same dominant strategy as in $g^{1}$, while neither strategy for player 2 is a dominant strategy but either is undominated.

\begin{tabular}{ccccccc}
\multicolumn{2}{c}{ Table 1. $g^{1}=\left(g_{1}^{1}, g_{2}^{1}\right)$} & & \multicolumn{3}{c}{ Table 2. $g^{2}=\left(g_{1}^{2}, g_{2}^{2}\right)$} \\
\cline { 6 - 7 } & $\mathbf{S}_{21}$ & $\mathbf{S}_{22}$ & & & $\mathbf{S}_{21}$ & $\mathbf{S}_{22}$ \\
\hline $\mathbf{s}_{11}$ & $(5,5)$ & $(1,6)$ & & $\mathbf{s}_{11}$ & $(5,5)$ & $(1,2)$ \\
$\mathbf{s}_{12}$ & $(6,1)$ & $(3,3)^{*}$ & & $\mathbf{s}_{12}$ & $(6,1)$ & $(3,3)^{*}$
\end{tabular}

For a comparison purpose, we mention Nash equilibrium. A strategy profile $s=\left(s_{1}, \ldots, s_{n}\right)$ is called a Nash equilibrium iff $s_{i}$ is a best strategy to $s_{-i}$ for all $i \in N$. In either of games $g^{1}$ and $g^{2},\left(\mathbf{s}_{12}, \mathbf{s}_{22}\right)$ is a unique Nash equilibrium. A Nash equilibrium in each matrix is marked with asterisk $*$.

In the following, we will use the following prediction-decision criteria to exemplify our theory.

DC1 (Dominant strategy): Player $i$ should choose a dominant strategy.

In game $g^{1}$, this criterion gives a decision to either player. In game $g^{2}$, however, DC1 gives a decision only to player 1 but not to player 2 , since player 2 has no dominant strategies. Note that this criterion includes no predictions, i.e., a player does not think about the other's choice. We may think that the lack of predictions causes the incapability of DC1 to recommend a decision for player 2. 
We consider another decision criterion including prediction-making.

DC2 (Best strategy to a dominant strategy): Player $i$ first predicts what the other player $j$ should choose following DC1, and then $i$ should choose a best strategy to the predicted strategy for $j$.

In game $g^{2}$, player 2 first predicts that 1 would choose $\mathbf{s}_{12}$ following DC1, and then 2 would choose $\mathbf{S}_{22}$ as the best strategy to the predicted decision $\mathbf{s}_{12}$. These criteria, DC1 and DC2, are related to the procedure of iterated elimination of dominated strategies (cf., Moulin [10] and Myerson [11]). These are more or less a standard example of a prediction-decision criterion in the literature of game theory. However, we can consider some other prediction-decision criteria which have never been discussed in the literature of game theory. We would like to show that our logical approach enables us to take such other prediction-decision criteria in its scope. In particular, we discuss the subtlety of inferential epistemic interactions required for decision making with such prediction-decision criteria.

Criterion DC2 makes no recommendations in some other games. For example, the games $g^{3}$ and $g^{4}$ (Matching Pennies) of Tables 3 and 4 allow neither player to have a dominant strategy. Neither DC1 nor DC2 makes a recommendation. However, some other criteria make recommendations for such games. Here we mention a few more decision criteria.

\begin{tabular}{cccccccc}
\multicolumn{3}{c}{ Table 3. $g^{3}=\left(g_{1}^{3}, g_{2}^{3}\right)$} & & \multicolumn{3}{c}{ Table 4. $g^{4}=\left(g_{1}^{4}, g_{2}^{4}\right)$} \\
\cline { 1 - 2 } \cline { 6 - 8 } & $\mathbf{s}_{21}$ & $\mathbf{s}_{22}$ & $\mathbf{s}_{23}$ & & & $\mathbf{s}_{21}$ & $\mathbf{s}_{22}$ \\
\hline $\mathbf{s}_{11}$ & $(5,5)$ & $(1,2)$ & $(4,3)$ & & $\mathbf{s}_{11}$ & $(1,-1)$ & $(-1,1)$ \\
$\mathbf{s}_{12}$ & $(6,1)$ & $(3,3)^{*}$ & $(0,2)$ & & $\mathbf{s}_{12}$ & $(-1,1)$ & $(1,-1)$ \\
& & & & & & & \\
\hline
\end{tabular}

As mentioned in Section 1, we treat "rationality" as an attribute of reasoning abilities rather than outcomes or behavior. In the present context, this mean that we do not pursue "rationality" of prediction-decision criteria. The following extreme example may clarify our attitude.

DC0 (Pure default decision): Player $i$ should choose the prespecified default strategy, e.g., $\mathbf{s}_{i 1}$.

In game $g^{4}$, player 1 can choose his first strategy $\mathbf{s}_{11}$ as a default decision with no further considerations. The word "default" has the connotation that after some other possibilities are considered and none of them recommends a decision, a default decision would be applied. We call this type the last-resort default decision criterion, which will be discussed in Section 9. Here, we treat a pure default decision just as a prespecified one.

Criterion DC0 may sound too trivial if it is applied to player $i$ 's own decision making. However, it would not be so if this is adopted for player $j$ 's prediction on $i$ 's decision making. That is, the prediction on the other player's choice is made without considerations on the other's subjective elements. We modify criterion DC2 into the following. 
DC20 (Dogmatic prediction decision): Player $i$ first predicts that player $j$ would choose strategy $\mathbf{s}_{j 1}$ following DC0, and then $i$ should choose a best strategy to the predicted strategy $\mathbf{s}_{i 1}$.

This criterion is free from the symmetric assumption that a decision maker assumes payoff maximization for himself as well as for the other player. In this sense, DC20 differs considerably from DC2.

We will see in Section 7 that criterion DC20 involves some subtlety in epistemic interactions, while DC0 has only trivial interactions.

Finally, we mention the very first decision making criterion considered by von Neumann [12] and [13] in the literature of game theory. We state it for player 1 .

vN (Maximin decision): Player 1 should choose his $s_{1}$ to $\operatorname{maximize}_{\min _{s_{2}}} g_{1}\left(s_{1}, s_{2}\right)$.

That is, player 1 evaluates each $s_{1}$ by the worst possible payoff, $\min _{s_{2}} g_{1}\left(s_{1}, s_{2}\right)$, and maximizes this value. This criterion is usually considered only for a twoperson zero-sum game. It is sometimes confused with a Nash equilibrium, since the resulting pair given by this criterion for two players is equivalent to a saddle point, i.e., Nash equilibrium, if it ever exists. However, $\mathrm{vN}$ is an individual decision criterion, and does not involve predictions about the other player's decision making. From the viewpoint of epistemic depth, this criterion has the same status as that of the dominant strategy criterion DC1. On the other hand, if we look at the saddle point in the game, and if we require the infinite regress argument such as in Kaneko [4], it would require common knowledge, i.e., the infinite depth. ${ }^{1}$

\subsection{Location game LG with three-stores of different sizes}

To exemplify our theory, we need a slightly more complex example. Consider the following 3-person game $h=\left(h_{1}, h_{2}, h_{3}\right)$. In the following, we call this 3-person game the location game LG. In LG, player 1 has two strategies $\mathbf{s}_{11}, \mathbf{s}_{12}$, and each $i=2,3$ has three strategies $\mathbf{s}_{i 1}, \mathbf{s}_{i 2}, \mathbf{s}_{i 3}$. We assume that $h_{1}$ depends upon all the three players' choices, $h_{2}$ depends upon his and 3's choice, and $h_{3}$ is determined solely by his own choice. Specifically, the payoff function $h_{1}\left(s_{1}, s_{2}, s_{3}\right)$ is given as

$$
h_{1}\left(s_{1}, s_{2}, s_{3}\right)=\left\{\begin{array}{cl}
2 & \text { if } s_{1}=\mathbf{s}_{11} \text { and } s_{2}=\mathbf{s}_{21} \\
1 & \text { if } s_{1}=\mathbf{s}_{11}, s_{2}=\mathbf{s}_{22} \text { and } s_{3} \neq \mathbf{s}_{31} \\
-1 & \text { if } s_{1}=\mathbf{s}_{11}, s_{2} \neq \mathbf{s}_{21} \text { and } s_{3}=\mathbf{s}_{31} \\
0 & \text { otherwise }
\end{array}\right.
$$

\footnotetext{
${ }^{1}$ Aumann and Maschler [1] discussed carefully the conceptual differences between the maximin decision criterion from the saddle point property (Nash equilibrium) from a different point of view from ours.
} 
and $h_{2}\left(s_{2}, s_{3}\right), h_{3}\left(s_{3}\right)$ are given as

$\begin{array}{lllllll} & \mathbf{s}_{31} & \mathbf{s}_{32} & \mathbf{s}_{33} & & \\ & & & & & \\ \mathbf{s}_{21} & 0 & 5 & 5 & \mathbf{s}_{31} & 10 \\ \mathbf{s}_{22} & 10 & 1 & 10 & \mathbf{s}_{32} & 20 \\ \mathbf{s}_{23} & 0 & 0 & 0 & \mathbf{s}_{33} & 0 \\ & & & & \\ & h_{2}\left(s_{2}, s_{3}\right) & & h_{3}\left(s_{3}\right)\end{array}$

Since this example is constructed to show some slightly complex epistemic interpersonal introspections, the following story in terms of industrial organizations looks somewhat twisted, but gives some idea on the scope of possible applications of our theory.

Three players 1,2,3 are companies, and there are three locations $a, b, c$ for these companies, which correspond to the three strategies $\mathbf{s}_{i 1}, \mathbf{s}_{i 2}, \mathbf{s}_{i 3}$ of player $i=2,3$. The demand at location $b$ is large, that at $a$ is medium, and that at $c$ is small. Player 3 is a large company treating many products, 2 a more specialized medium company, and 1 a small company specialized to one product. Player 1 has already a facility at location $a$, and then his choice is to open, $\mathbf{s}_{11}$, or not to open a store, $\mathbf{s}_{12}$ at location $a$. Players 2 and 3 would choose one location to open a store. Player 1's product is complemental to the those treated by player 2. Therefore, player 1 wants player 2 to open a store at location $a$. If player 2 chooses $a$, then 1 would have profit 2 . If 2 opens at location $b$, then 1 would get profit 1 unless the big store 3 comes to location $a$. It is the worst case for 1 that 1 opens the store and only the big store 3 comes to $a$. Player 2 is affected by player 3's choice. However, 3 is large enough to ignore the other two players. ${ }^{2}$

Since player 1 is affected by the other two players, player 1's decision making may involve new aspects not found in the prediction-decision criteria described in Section 2.1. Therefore, we consider only player 1's decision making in the location game LG.

The following one is an extension of DC2 to the 3-player case.

HDC (Hierarchal decision criterion): Player 1 predicts that 2 would predict what 3 would choose following DC1, and that 2 would choose the best strategy to it. Then player 1 should choose a best strategy to his prediction on 2's decision.

Specifically, player 1 predicts that 2 predicts that 3 would choose $\mathbf{s}_{32}$ as the dominant strategy, and then 1 would predict that 2 would choose $\mathbf{s}_{21}$ as the best strategy to $\mathbf{s}_{32}$. Then player 1 would choose $\mathbf{s}_{11}$ as the best strategy to the prediction $\mathbf{s}_{21}$. Thus, player 1 can make a decision by this criterion. In this case, player 1 needs to think about 2's decision making as well as 2' prediction about 3's decision making. However, 1 does not directly predict 3's decision making, and his prediction is made only through 2's mind.

\footnotetext{
2 Our concern is ordinal preferences on strategy profiles. This assumption can be interpreted as meaning that the ordinal preferences are determined by $h_{2}\left(s_{2}, s_{3}\right)$ and $h_{3}\left(s_{3}\right)$, though the actual profits are slightly influenced by the choices of 1 and/or of 2 .
} 
In the present location game, player 1 can make a decision under the prediction that the other players choose undominated strategies.

NPC (Negative prediction criterion): Player 1 predicts that 2 and 3 would choose undominated strategies, and 1 would choose a best strategy to his predictions.

Specifically in the above game, player 1 predicts that players 2 and 3 would choose, respectively, $\mathbf{s}_{21}$ or $\mathbf{s}_{22}$ and $\mathbf{s}_{32}$ as undominated strategies. Then he should choose $\mathbf{s}_{11}$ as a best strategy to either of $\left(\mathbf{s}_{21}, \mathbf{s}_{32}\right)$ and $\left(\mathbf{s}_{22}, \mathbf{s}_{32}\right)$. In this case, player 1 thinks about 2's decision making only in a negative sense, i.e., what players 2 and 3 would not choose. In location game LG, player 1 can also make a decision by this criterion.

The prediction-decision criteria HDC and NPC require different interpersonal introspections. In HDC, the interpersonal introspection takes a liner form: 1 thinks about 2's prediction about 3's decision. On the other hand, in NPC, the interpersonal introspection takes a branching form: 1 thinks about 2's and 3's decisions separately. This difference will be more explicitly discussed in Sections 7,8 and 9 .

\section{Set of formulae $\mathscr{P}$ and epistemic structures}

We define the set of formulae and see how prediction-decision criteria are described as formulae. We also define the concept of an epistemic structure, which will be used in descriptive and inferential manners for the definition of our epistemic logic GL $\mathrm{EF}_{\mathrm{F}}$ of shallow depths in Section 4.

\subsection{Definition of formulae}

We represent payoff functions $g_{1}, \ldots, g_{n}$ in terms of preference relations. We start with:

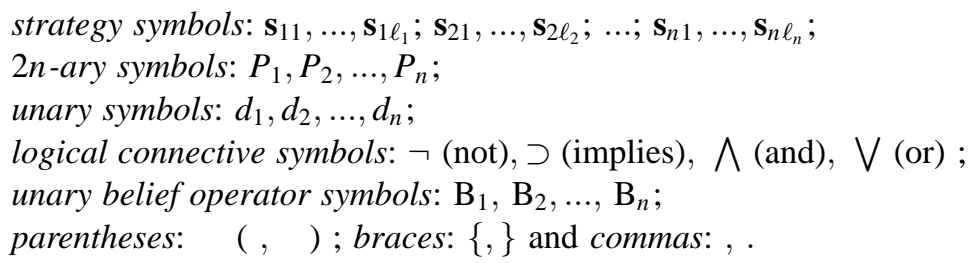

We associate the intended meanings, "not", "implies", "and", "or", with connective symbols, $\neg, \supset, \bigwedge, \bigvee$, respectively. Unary belief operator symbol $\mathrm{B}_{i}$ is applied to each formula. Strategy symbols are identical to those given in the Section 2 . By a $2 n$-ary symbol $P_{i}$, we consider the expression $P_{i}\left(s_{1}, \ldots, s_{n}: t_{1}, \ldots, t_{n}\right)$ for $\left(s_{1}, \ldots, s_{n}\right),\left(t_{1}, \ldots, t_{n}\right) \in S$. By a unary symbol $d_{i}$, we consider the expression $d_{i}\left(s_{i}\right)$ for $s_{i} \in S_{i}$. These expressions are called atomic formulae, and the set of them is denoted by $A F$. For example, when $n=2$ and $\ell_{1}=\ell_{2}=2, A F$ consists 
of $32+4$ atomic formulae. Atomic formula $P_{i}\left(s_{1}, \ldots, s_{n}: t_{1}, \ldots, t_{n}\right)$ is intended to means a weak preference for $\left(s_{1}, \ldots, s_{n}\right)$ over $\left(t_{1}, \ldots, t_{n}\right)$ for player $i$, and $d_{i}\left(s_{i}\right)$ means that $s_{i}$ is a default decision for player $i$.

Regarding $A F$ as the set of propositional variables, we define formulae inductively as follows:

F1: any $A \in A F$ is a formula;

F2: if $A$ and $B$ are formulae, so are $(\neg A),(A \supset B)$ and $\mathrm{B}_{i}(A)(i \in N)$;

F3: if $\left\{A_{0}, A_{1}, \ldots, A_{m}\right\}$ is a finite nonempty set of formulae, then $\left(\bigwedge\left\{A_{0}, A_{1}, \ldots\right.\right.$, $\left.\left.A_{m}\right\}\right)$ and $\left(\bigvee\left\{A_{0}, A_{1}, \ldots, A_{m}\right\}\right)$ are also formulae;

F4: any formula is obtained by a finite number of applications of F1, F2 and F3. ${ }^{3}$

We denote the set of all formulae by $\mathscr{P}$. We say that a formula $A$ is nonepistemic iff $A$ contains no $\mathrm{B}_{1}, \ldots, \mathrm{B}_{n}$. We denote the set of all nonepistemic formulae by $\mathscr{P}^{\mathrm{n}}$. We follow standard practices of abbreviations so that we could recover the original expressions when necessary. We will also abbreviate $\bigwedge\{A, B\}, \bigvee\{A, B, C\}, \bigwedge\left\{A_{0}, A_{1}, \ldots, A_{m}\right\}$ as $A \wedge B, A \vee B \vee C, \bigwedge_{k=0}^{m} A_{k}$, etc. We denote $(A \supset B) \wedge(B \supset A)$ by $A \equiv B$. We also denote $\neg p \wedge p$ and $\neg p \vee p$ by $\perp$ and $\top$, respectively, where $p$ is an atomic formula.

Here we look briefly at how the basic game theoretical concepts are expressed in our language.

First, we express the payoff function $g_{i}$ of player $i$ as the following set of preferences:

$$
\left\{P_{i}(s: t): g_{i}(s) \geq g_{i}(t)\right\} \cup\left\{\neg P_{i}(s: t): g_{i}(s)<g_{i}(t)\right\},
$$

which is denoted by $\hat{g}_{i}$. The conjunction $\bigwedge \hat{g}_{i}$ of $\hat{g}_{i}$ is a formula. Hence, the payoff functions for $n$ players are described as the set $\hat{g}=\hat{g}_{1} \cup \cdots \cup \hat{g}_{n}$ or as the formula $\bigwedge\left(\hat{g}_{1} \cup \cdots \cup \hat{g}_{n}\right)$.

The statement that strategy $s_{i}$ for player $i$ is a best strategy to the others' strategies $s_{-i}$ is described as the formula $\bigwedge\left\{P_{i}\left(s_{i} ; s_{-i}: t_{i} ; s_{-i}\right): t_{i} \in S_{i}\right\}$, which we denote by $\operatorname{Best}_{i}\left(s_{i} \mid s_{-i}\right)$. The statement that $s_{i}$ is a dominant strategy for player $i$ is expressed as $\bigwedge\left\{\operatorname{Best}_{i}\left(s_{i} \mid s_{-i}\right): s_{-i} \in S_{-i}\right\}$. This means that $s_{i}$ is the most preferable whatever the others choose. This is equivalent to $\bigwedge\left\{P_{i}\left(s_{i} ; t_{-i}\right.\right.$ : $\left.t_{i} ; t_{-i}\right): t_{i} \in S_{i}$ and $\left.t_{-i} \in S_{-i}\right\}$, which we denote by $\operatorname{Dom}_{i}\left(s_{i}\right)$.

An undominated strategy needs a slightly longer definition: First, we denote the following by $\operatorname{dom}_{i}\left(t_{i}, s_{i}\right)$ :

$$
\bigwedge_{s_{-i} \in S_{-i}} P_{i}\left(t_{i} ; s_{-i}: s_{i} ; s_{-i}\right) \wedge \bigvee_{s_{-i} \in S_{-i}} \neg P_{i}\left(s_{i} ; s_{-i}: t_{i} ; s_{-i}\right)
$$

\footnotetext{
${ }^{3}$ The above definition deviates from the standard textbook definition of formulae in that connectives $\bigwedge$ and $\bigvee$ are applied to a finite nonempty set of formulae, e.g., $\bigwedge\left\{A_{0}, A_{1}, \ldots, A_{m}\right\}$, rather than to an ordered pair of formulae. We take this deviation to facilitate game theoretical applications. However, the resulting logical systems are equivalent (with respective to provabilities or validities defined in the systems). This formulation does not fit in some considerations, e.g., using the Gödel numbering. If one wants to take the Gödel numbering, then the standard formulation would be more convenient.
} 
The above, (3.2), states " $t_{i}$ dominates $s_{i}$ ". Using this formula, we define $\operatorname{Und}_{i}\left(s_{i}\right):=\bigwedge_{t_{i}} \neg \operatorname{dom}_{i}\left(t_{i}, s_{i}\right)$. This states " $s_{i}$ is an undominated strategy".

Finally, consider the prediction-decision criteria DC1 and DC2 for a 2-person game $g=\left(g_{1}, g_{2}\right)$. Criterion DC1 for player $i$ is described as $\mathscr{D}_{i}^{1}=\left\{\operatorname{Dom}_{i}\left(s_{i}\right)\right.$ : $\left.s_{i} \in S_{i}\right\}$. Criterion DC2 for player $i$ is denoted by $\mathscr{D}_{i}^{2}=\left\{D_{i}^{2}\left(s_{i}\right): s_{i} \in S_{i}\right\}$, where each $D_{i}^{2}\left(s_{i}\right)$ is given as

$$
\bigvee_{s_{j}} \mathrm{~B}_{j}\left(\operatorname{Dom}_{j}\left(s_{j}\right)\right) \wedge \bigwedge_{s_{j}}\left(\mathrm{~B}_{j}\left(\operatorname{Dom}_{j}\left(s_{j}\right)\right) \supset \operatorname{Best}_{i}\left(s_{i} \mid s_{j}\right)\right) .
$$

If player $i$ believes $\Gamma$ and if $\mathrm{B}_{i}\left(D_{i}^{2}\left(s_{i}\right)\right)$ is derived from his beliefs $\mathrm{B}_{i}(\Gamma)$, then $s_{i}$ is regarded as a decision for $i$ as far as he adopts his prediction-decision criterion $\mathscr{D}_{i}^{2}$.

In Section 5, we discuss these and other prediction-decision criteria in a unified manner.

\subsection{Epistemic depths of formulae and epistemic structures}

Although the set of formulae $\mathscr{P}$ allows any finitely nested structures of $\mathrm{B}_{1}, \ldots$, $\mathrm{B}_{n}$, the decision criteria $\mathscr{D}_{i}^{1}$ and $\mathscr{D}_{i}^{2}$ seem to need only small part of $\mathscr{P}$. To capture this idea, we introduce the notions of the epistemic depths of formulae and epistemic structures. As stated in Section 1, the notion of epistemic structures will be used to impose restrictions on:

(i): interpersonal epistemic expressions in formulae;

(ii): interpersonal epistemic inferences in proofs.

In this section, we will discuss only (i), and will do (ii) in Section 4.

First, let $N^{<\omega}:=\left\{\left(i_{1}, \ldots, i_{m}\right): i_{1}, \ldots, i_{m} \in N\right\}$. Note that $N^{<\omega}$ contains the null sequence $\epsilon$, i.e., the sequence of length 0 . We call $e=\left(i_{1}, \ldots, i_{m}\right) \in N^{<\omega}$ an epistemic status. For $e=\left(i_{1}, \ldots, i_{m}\right) \in N^{<\omega}, \mathrm{B}_{i_{1}} \ldots \mathrm{B}_{i_{m}}(A)$ is denoted by $\mathrm{B}_{e}(A)$, and $\mathrm{B}_{\epsilon}(A)$ is regarded as $A$. We define the following concatenation: for $e=\left(i_{1}, \ldots, i_{m}\right)$, $e^{\prime}=\left(j_{1}, \ldots, j_{k}\right) \in N^{<\omega}$, let $e \circ e^{\prime}=\left(i_{1}, \ldots, i_{m}, j_{1}, \ldots, j_{k}\right)$. We also let $e \circ \epsilon=\epsilon \circ e=e$. We write $(i) \circ e$ and $e \circ(i)$ as $i \circ e$ and $e \circ i$, respectively.

We define the (epistemic) depth $\delta^{r}(A)$ of $A \in \mathscr{P}$ by induction on the length of a formula:

D0: $\delta^{r}(p)=\{\epsilon\}$ for any $p \in A F$;

D1: $\delta^{r}(\neg C)=\delta^{r}(C)$;

D2: $\delta^{r}(C \supset D)=\delta^{r}(C) \cup \delta^{r}(D)$;

D3: $\delta^{r}(\bigwedge \Phi)=\delta^{r}(\bigvee \Phi)=\bigcup_{C \in \Phi} \delta^{r}(C)$;

D4: $\delta^{r}\left(\mathrm{~B}_{i}(C)\right)=\left\{i \circ e: e \in \delta^{r}(C)\right\}$.

Note that $\delta^{r}(A)$ is a subset of $N^{<\omega}$. For example, $\delta^{r}\left(\mathrm{~B}_{2}\left(\operatorname{Dom}_{2}\left(s_{2}\right)\right)\right)=\{(2)\}$ and 
$\delta^{r}\left(\mathrm{~B}_{2}\left(D_{2}^{2}\left(s_{2}\right)\right)\right)=\{(2),(2,1)\}$. We define $\delta^{r}(\Gamma)=\bigcup_{C \in \Gamma} \delta^{r}(C)$ for a set $\Gamma$ of formulae. $^{4}$

To give a restriction on formal descriptions and formal proofs, we introduce the notion of an epistemic structure. We say that a nonempty subset $E$ of $N^{<\omega}$ is an epistemic structure iff

$$
\left(i_{1}, \ldots, i_{m}\right) \in E \text { implies }\left(i_{1}, \ldots, i_{m-1}\right) \in E .
$$

When $m=1,\left(i_{1}, \ldots, i_{m-1}\right)$ is the null symbol $\epsilon$. By the nonemptiness of $E$ and (3.4), we have $\epsilon \in E$. Trivial examples for $E$ are $N^{<\omega}$ and $\{\epsilon\}$. Less trivial examples are $\{\epsilon,(1),(2)\}$ and $\{\epsilon,(1),(2),(1,2),(2,1)\}$. The epistemic depth $\delta^{r}(A)$ of formula $A$ may not satisfy (3.4). However, for any given $A$, there is the smallest epistemic structure including $\delta^{r}(A)$.

Given an epistemic structure $E$, we define

$$
\mathscr{P} E=\left\{A \in \mathscr{P}: \delta^{r}(A) \subseteq E\right\} .
$$

A formula $A$ in $\mathscr{T} E$ is said to be admissible in $E$. That is, when an (descriptive) epistemic structure $E$ is given, we admit only formulae whose depths are in $E$. For example, when $E=\{\epsilon,(1),(2)\}$, any formula in $\mathscr{T}_{E}$ may have $\mathrm{B}_{1}$ and $\mathrm{B}_{2}$ without nested occurrences. On the other hand, formula $\mathrm{B}_{2}\left(D_{2}^{2}\left(s_{2}\right)\right)$ belongs to $\mathscr{P}_{\{\epsilon,(2),(2,1)\}}$ but not to $\mathscr{P}_{E}$. Since the null symbol $\epsilon$ always belongs to any epistemic structure $E$, all the nonepistemic formulae are included in $\mathscr{P}_{E}$, i.e., $\mathscr{P}^{\mathrm{n}} \subseteq \mathscr{P}_{E}$

\section{Epistemic logic $G_{E F}$ of shallow depths}

We adopt the Gentzen-style formulation of the epistemic logic $\mathrm{GL}_{E F}$ of shallow depths. The choice of the Gentzen-style is made so as to facilitate our arguments faithfully. Intuitively speaking, $\mathrm{GL}_{E F}$ is defined by imposing two restrictions on formulae and proofs in the Gentzen-style formulation of $\mathrm{KD}^{n}$. In Section 4.1, we give the Gentzen formulation of $\mathrm{GL}_{E F}$, and in Section 4.2, we see how it is used for describing game theoretic decision making. We give detailed examples and explanations in Section 4.3 and also state basic theorems on logic $\mathrm{GL}_{E F}$ in Section 4.4.

\subsection{Logic $G L_{E F}$ and game theoretic statements}

Let $E$ and $F$ be two epistemic structures with $F \subseteq E$. We give restrictions in terms of these $E$ and $F$, respectively, on formulae and on proofs. To formulate the restrictions on proofs, we introduce the concept of a thought sequent.

Let $e=\left(i_{1}, \ldots, i_{m}\right) \in E$, and $\Gamma, \Theta$ finite subsets of $\mathscr{P}_{E}$. Using auxiliary symbols [, ], and $\rightarrow$, we introduce a new expression $\mathrm{B}_{e}[\Gamma \rightarrow \Theta]:=\mathrm{B}_{i_{1}} \ldots \mathrm{B}_{i_{m}}[\Gamma \rightarrow$

\footnotetext{
${ }^{4}$ This $\delta^{r}$ differs in D4 from $\delta$ given in Kaneko [4], which is the depth measure suited to the KD4-type logics.
} 
$\Theta$ ], which we call a thought sequent. The admissibility of a formula is extended to a thought sequent. We say that a thought sequent $\mathrm{B}_{e}[\Gamma \rightarrow \Theta]$ is admissible in $E$ iff $e \circ \delta(\Gamma \cup \Theta):=\left\{e \circ e^{\prime}: e^{\prime} \in \delta(\Gamma \cup \Theta)\right\} \subseteq E$. Admissible proofs with respect to $F$ will be defined presently.

We abbreviate $\mathrm{B}_{\epsilon}[\Gamma \rightarrow \Theta]$ as $\Gamma \rightarrow \Theta$. Also, we abbreviate $\mathrm{B}_{e}[\Gamma \cup \Delta \rightarrow$ $\Lambda \cup \Theta]$ and $\mathrm{B}_{e}[\{A\} \cup \Gamma \rightarrow \Theta \cup\{C\}]$ as $\mathrm{B}_{e}[\Gamma, \Delta \rightarrow \Lambda, \Theta]$ and $\mathrm{B}_{e}[A, \Gamma \rightarrow \Theta, C]$, etc. We use the convention to write $\mathrm{B}_{i}(\Phi)=\left\{\mathrm{B}_{i}(A): A \in \Phi\right\}$.

The notion of a thought sequent has some conceptual difference from Gentzen's [3] original notion of a sequent. Nevertheless, since we consider only thought sequents, we may call thought sequents simply sequents.

By $\mathrm{B}_{i_{1}} \ldots \mathrm{B}_{i_{m}}[\Gamma \rightarrow \Theta]$, we express the idea that player $i_{m}$ in the mind of $i_{m-1}$ $\ldots$ in the mind of $i_{1}$ conducts logical reasoning and believes that $\Gamma \rightarrow \Theta$. As in the standard sequent calculus, $\Gamma \rightarrow \Theta$ is intended to mean $\wedge \Gamma \supset \bigvee \Theta$, where $\wedge \emptyset$ and $\bigvee \emptyset$ are meant to be $\neg p \vee p$ and $\neg p \wedge p$, respectively. Here, we note that if we forget the outer $\mathrm{B}_{e}[\cdots]$ of $\mathrm{B}_{e}[\Gamma \rightarrow \Theta]$ and impose no restrictions on formulae and proofs, the following logical system would be the same as the Gentzen-style formulation of $\mathrm{KD}^{n}$.

The logical reasoning of the innermost player $i_{m}$ in $\mathrm{B}_{i_{1}} \ldots \mathrm{B}_{i_{m}}[\cdots]$ is governed by one axiom schema and various inference rules, which describe classical logic. One additional rule connects player $i_{m}$ 's reasoning to $i_{m-1}$ 's. In the following, $\Gamma, \Theta, \Delta, \Lambda, \Phi$ are finite sets of formulae, $A, B$ formulae and $\Phi$ is assumed to be nonempty.

Axiom (Initial Sequent): $\mathrm{B}_{e}[A \rightarrow A]$,

\section{Structural Rules:}

$$
\frac{\mathrm{B}_{e}[\Gamma \rightarrow \Theta]}{\mathrm{B}_{e}[\Delta, \Gamma \rightarrow \Theta, \Lambda]}(\mathrm{Th}) \quad \frac{\mathrm{B}_{e}[\Gamma \rightarrow \Theta, A] \quad \mathrm{B}_{e}[A, \Delta \rightarrow \Lambda]}{\mathrm{B}_{e}[\Gamma, \Delta \rightarrow \Theta, \Lambda]} \text { (Cut) }
$$

\section{Operational Rules:}

$$
\begin{aligned}
& \frac{\mathrm{B}_{e}[\Gamma \rightarrow \Theta, A]}{\mathrm{B}_{e}[\neg A, \Gamma \rightarrow \Theta]}(\neg \rightarrow) \quad \frac{\mathrm{B}_{e}[A, \Gamma \rightarrow \Theta]}{\mathrm{B}_{e}[\Gamma \rightarrow \Theta, \neg A]}(\rightarrow \neg) \\
& \frac{\mathrm{B}_{e}[\Gamma \rightarrow \Theta, A] \quad \mathrm{B}_{e}[B, \Gamma \rightarrow \Theta]}{\mathrm{B}_{e}[A \supset B, \Gamma \rightarrow \Theta]}(\supset \rightarrow) \quad \frac{\mathrm{B}_{e}[A, \Gamma \rightarrow \Theta, B]}{\mathrm{B}_{e}[\Gamma \rightarrow \Theta, A \supset B]}(\rightarrow \supset) \\
& \frac{\mathrm{B}_{e}[A, \Gamma \rightarrow \Theta]}{\mathrm{B}_{e}[\bigwedge \Phi, \Gamma \rightarrow \Theta]}(\bigwedge \rightarrow), \text { where } A \in \Phi \quad \frac{\left\{\mathrm{B}_{e}[\Gamma \rightarrow \Theta, A]: A \in \Phi\right\}}{\mathrm{B}_{e}[\Gamma \rightarrow \Theta, \bigwedge \Phi]}(\rightarrow \bigwedge) \\
& \frac{\left\{\mathrm{B}_{e}[A, \Gamma \rightarrow \Theta]: A \in \Phi\right\}}{\mathrm{B}_{e}[\bigvee \Phi, \Gamma \rightarrow \Theta]}(\bigvee \rightarrow) \quad \frac{\mathrm{B}_{e}[\Gamma \rightarrow \Theta, A]}{\mathrm{B}_{e}[\Gamma \rightarrow \Theta, \bigvee \Phi]}(\rightarrow \bigvee) \text {, where } A \in \Phi
\end{aligned}
$$

Epistemic Distribution Rule:

$$
\frac{\mathrm{B}_{e \circ i}[\Gamma \rightarrow \Theta]}{\mathrm{B}_{e}\left[\mathrm{~B}_{i}(\Gamma) \rightarrow \mathrm{B}_{i}(\Theta)\right]}\left(\mathrm{B}_{i} \rightarrow \mathrm{B}_{i}\right) \text {, where }|\Theta| \leq 1 \text { and } i \in N \text {. }
$$


Here $|\Theta|$ is the cardinality of $\Theta$.

Inferences $(\supset \rightarrow),(\rightarrow \wedge)$ and $(\bigvee \rightarrow)$ have the sets of upper (thought) sequents, which mean that each sequent is already proved. Some examples are given below.

The outer $\mathrm{B}_{e}[\cdots]$ of the upper and lower thought sequents in each of the structural and operational rules are identical, and these rules describe classic logic. That is, the innermost player $i_{m}$ in $e=\left(i_{1}, \ldots, i_{m}\right)$ is assumed to be capable of conducting logical reasonings described by classical logic. The outer $\mathrm{B}_{e}[\cdots]$ changes only at $\left(\mathrm{B}_{i_{m}} \rightarrow \mathrm{B}_{i_{m}}\right)$, and eventually the innermost $i_{m}$ goes into the scope of $\mathrm{B}_{\left(i_{1}, \ldots, i_{m-1}\right)}[\cdots]$. The length of $e=\left(i_{1}, \ldots, i_{m}\right)$ of the outer $\mathrm{B}_{e}[\cdots]$ gets shorter only at an application of $\left(\mathrm{B}_{i_{m}} \rightarrow \mathrm{B}_{i_{m}}\right)$ in a proof.

Let $\mathrm{B}_{e}[\Gamma \rightarrow \Theta]$ be a thought sequent admissible in $E$. An admissible proof $P$ of $\mathrm{B}_{e}[\Gamma \rightarrow \Theta]$ in $\mathrm{GL}_{E F}$ is a finite tree satisfying the following conditions:

P1: a thought sequent admissible in $E$ is associated with each node;

P2: the thought sequent associated with each leaf is an instance of the axiom;

P3: adjoining nodes together with their associated thought sequents form an instance of the above inference rules;

P4: $\mathrm{B}_{e}[\Gamma \rightarrow \Theta]$ is associated with the root node;

P5: $e^{\prime}$ belongs to $F$ for any thought sequent $\mathrm{B}_{e^{\prime}}[\Delta \rightarrow \Lambda]$ in $P$.

We say that $\mathrm{B}_{e}[\Gamma \rightarrow \Theta]$ is provable in $\mathrm{GL}_{E F}$, denoted by $\vdash_{E F} \mathrm{~B}_{e}[\Gamma \rightarrow \Theta]$, iff there is an admissible proof $P$ of $\mathrm{B}_{e}[\Gamma \rightarrow \Theta]$ in $\mathrm{GL}_{E F}$. The negation of $\vdash_{E F} \mathrm{~B}_{e}[\Gamma \rightarrow \Theta]$ is denoted by $\nvdash_{E F} \mathrm{~B}_{e}[\Gamma \rightarrow \Theta]$. Recall that when $e=\epsilon$, we abbreviate the outer $\mathrm{B}_{\epsilon}[\cdots]$, that is, $\vdash_{E F} \mathrm{~B}_{\epsilon}[\Gamma \rightarrow \Theta]$ is written as $\vdash_{E F} \Gamma \rightarrow \Theta$. When we write $\vdash_{E F} \Gamma \rightarrow \Theta$ or $\nvdash_{E F} \Gamma \rightarrow \Theta$, we already assume that $\Gamma \rightarrow \Theta$ is admissible in $E$, i.e., $\delta(\Gamma \cup \Theta) \subseteq E$.

Consider one example of a proof. In Kaneko [4], it is shown that $\mathrm{B}_{i}\left(\bigwedge \hat{g}_{i}^{1}\right) \supset$ $\mathrm{B}_{i}\left(\operatorname{Dom}_{i}\left(\mathbf{s}_{i 2}\right)\right)$ is provable in $\mathrm{KD}^{n}$. This is now proved for $i=1$ in $\mathrm{GL}_{E F}$ with $E=F=\{\epsilon,(1)\}$ as follows:

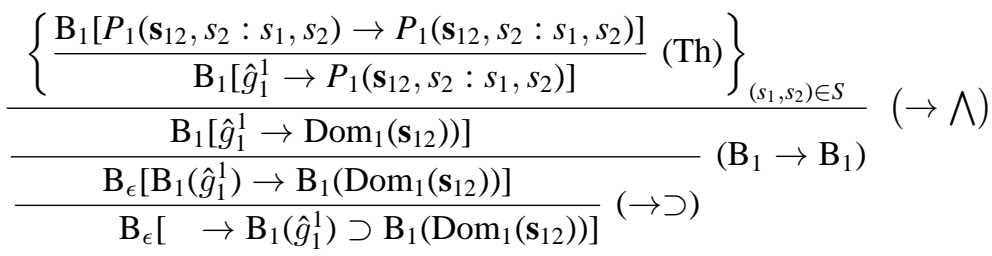

Note that inference $(\rightarrow \bigwedge$ ) has $|S|=2 \times 2$ upper thought sequents, each of which is derived with (Th). In fact, $\vdash_{E F} \rightarrow \mathrm{B}_{1}\left(\hat{g}_{1}^{1}\right) \supset \mathrm{B}_{1}\left(\operatorname{Dom}_{1}\left(\mathbf{s}_{12}\right)\right)$ is equivalent to $\vdash_{E F} \mathrm{~B}_{1}\left(\hat{g}_{1}^{1}\right) \rightarrow \mathrm{B}_{1}\left(\operatorname{Dom}_{1}\left(\mathbf{s}_{12}\right)\right)$.

We define the inconsistency of $\Gamma$ in $\mathrm{GL}_{E F}$ by $\vdash_{E F} \Gamma \rightarrow \perp$. Recall that $\perp$ is the formula $\neg p \wedge p$, where $p \in A F$. We say that $\Gamma$ is consistent in GL $_{E F}$ iff $\nvdash_{E F} \Gamma \rightarrow \perp$. We will use the following fact:

$$
\Gamma \text { is consistent in } \mathrm{GL}_{E F} \text { if and only if } \nvdash_{E F} \Gamma \rightarrow \text {. }
$$


Indeed, let $\vdash_{E F} \Gamma \rightarrow \perp$. First, we have $\vdash_{E F} \perp \rightarrow$, which is proved as follows:

$\begin{array}{cc}\frac{p \rightarrow p}{\neg p, p \rightarrow} & (\neg \rightarrow) \\ \frac{\neg p \wedge p, p \rightarrow}{\neg p \wedge p \rightarrow} & (\wedge \rightarrow) \\ & (\wedge \rightarrow),\end{array}$

Then we combine this proof with a proof of have $\Gamma \rightarrow \perp$ as follows:

$$
\frac{\frac{\cdot \cdot \cdot}{\Gamma \rightarrow \perp} \stackrel{\cdot \cdot \cdot}{\perp \rightarrow}}{\Gamma \rightarrow} \text { (Cut). }
$$

Conversely, if $\vdash_{E F} \Gamma \rightarrow$, then $\vdash_{E F} \Gamma \rightarrow \perp$ by (Th). Thus, we have (4.2).

Also, when $(i) \in F$, the following hold and will be used without mentioning:

$$
\vdash_{E F} \mathrm{~B}_{i}(A \wedge C) \rightarrow \mathrm{B}_{i}(A) \wedge \mathrm{B}_{i}(C) \text { and } \vdash_{E F} \mathrm{~B}_{i}(A) \wedge \mathrm{B}_{i}(C) \rightarrow \mathrm{B}_{i}(A \wedge C) .
$$

Note that for these, we use $\left(\mathrm{B}_{i} \rightarrow \mathrm{B}_{i}\right)$ once. This interchangeability holds for any finite nonempty set $\Phi$ of formulae, i.e., $\vdash_{E F} \mathrm{~B}_{i}(\bigwedge \Phi) \rightarrow \bigwedge \mathrm{B}_{i}(\Phi)$ and $\vdash_{E F}$ $\bigwedge \mathrm{B}_{i}(\Phi) \rightarrow \mathrm{B}_{i}(\bigwedge \Phi)$

We need more comments, examples and basic theorems so as to use the above Gentzen-style formulation of $\mathrm{GL}_{E F}$ for investigations of game theoretical decision making. We postpone such details to the next subsections, and here we mention only how these definitions are used to describe the general problem of game theoretical decision making.

\subsection{Statements on game theoretical decision making}

From the viewpoint of the investigator (observer), the problem is stated as the provability or unprovability of thought sequent

$$
\Gamma \rightarrow A \text {. }
$$

Here, $\Gamma$ is the set of formulae assumed by the investigator, and $A$ is a consequence to be derived from these assumptions $\Gamma$. Hence, if (4.3) is provable, we regard $A$ as derived from the assumptions $\Gamma$ by the investigator, but if not, we regard $A$ as underivable from $\Gamma$ by the investigator.

When the investigator thinks about the logical reasoning of player $i,(4.3)$ may be expressed as

$$
\mathrm{B}_{i}\left(\Gamma_{i}\right) \rightarrow \mathrm{B}_{i}\left(A_{i}\right)
$$

That is, $\Gamma$ and $A$ takes the forms of $\mathrm{B}_{i}\left(\Gamma_{i}\right)$ and $\mathrm{B}_{i}\left(A_{i}\right)$. Exactly speaking, this means that the investigator, rather than player $i$, has the assumptions $\mathrm{B}_{i}\left(\Gamma_{i}\right)$ and derives consequence $\mathrm{B}_{i}(A)$ from $\mathrm{B}_{i}\left(\Gamma_{i}\right)$. Conceptually, this differs from 


$$
\mathrm{B}_{i}\left[\Gamma_{i} \rightarrow A_{i}\right]
$$

This thought sequent means that player $i$ derives $A_{i}$ from his beliefs $\Gamma_{i}$. When this sequent is provable, the investigator understands rationally the derivability of $A_{i}$ from $\Gamma_{i}$ by player $i$. Here, the rationality is entirely in the sense of logic. Regardless of the conceptual difference, the provabilities of (4.4) and (4.5) are equivalent in $\mathrm{GL}_{E F}$ with $i \in F$. This will be proved in Section 6 .

Now, consider a prediction-decision criterion. A prediction-decision criterion is given as $\mathscr{D}_{i}=\left\{D_{i}\left(s_{i}\right): s_{i} \in S_{i}\right\}$, where $D_{i}\left(s_{i}\right)$ is a formula indexed by $s_{i}$. The criteria DC1 and DC2 given in Section 2.1 are examples for $\mathscr{D}_{i}$, that is, these are formulated as $\mathscr{D}_{i}{ }^{1}=\left\{\operatorname{Dom}_{i}\left(s_{i}\right): s_{i} \in S_{i}\right\}$ and $\mathscr{D}_{i}^{2}=\left\{\bigvee_{s_{j}} \mathrm{~B}_{j}\left(\operatorname{Dom}_{j}\left(s_{j}\right)\right) \wedge\right.$ $\left.\bigwedge_{s_{j}}\left(\mathrm{~B}_{j}\left(\operatorname{Dom}_{j}\left(s_{j}\right)\right) \supset \operatorname{Best}_{i}\left(s_{i} \mid s_{j}\right)\right): s_{i} \in S_{i}\right\}$.

The following thought sequent represents player $i$ 's capability of decision making:

$$
\mathrm{B}_{i}\left(\Gamma_{i}\right) \rightarrow \bigvee_{s_{i}} \mathrm{~B}_{i}\left(D_{i}\left(s_{i}\right)\right),
$$

where $\Gamma_{i}$ is the finite set of player $i$ 's basic beliefs. This means that the existence of some decision $s_{i}$ is derived from player $i$ 's beliefs $\Gamma_{i}$.

The general statement by the investigator is expressed as the provability of the following:

$$
\Gamma_{0}, \mathrm{~B}_{1}\left(\Gamma_{1}\right), \ldots, \mathrm{B}_{n}\left(\Gamma_{n}\right) \rightarrow \bigvee_{s_{1}} \mathrm{~B}_{1}\left(D_{1}\left(s_{1}\right)\right) \wedge \ldots \wedge \bigvee_{s_{n}} \mathrm{~B}_{n}\left(D_{n}\left(s_{n}\right)\right),
$$

where $\Gamma_{0}$ is a finite set of nonepistemic formulae, which expresses the objective situation such as $\hat{g}_{1} \cup \ldots \cup \hat{g}_{n}$. This is a statement on the capabilities of the decision making of all the players. ${ }^{5}$

We regard, as a goal of this paper, the consideration of the provability of (4.7) from the viewpoint of the investigator. We mention the following theorem, though its proof needs various meta-theorems and will be given in Section 6 . The theorem states that for each player's decision making, it suffices to consider (4.6) separately and to ignore the objective part $\Gamma_{0}$.

Theorem 4.1 (Decomposition). Suppose that $\Gamma_{0} \cup \mathrm{B}_{1}\left(\Gamma_{1}\right) \cup \ldots \cup \mathrm{B}_{n}\left(\Gamma_{n}\right)$ is consistent in $\mathrm{GL}_{E F}$. Then the following two statements are equivalent:

(1): $\vdash_{E F} \Gamma_{0}, \mathrm{~B}_{1}\left(\Gamma_{1}\right), \ldots, \mathrm{B}_{n}\left(\Gamma_{n}\right) \rightarrow \bigvee_{s_{1}} \mathrm{~B}_{1}\left(D_{1}\left(s_{1}\right)\right) \wedge \ldots \wedge \bigvee_{s_{n}} \mathrm{~B}_{n}\left(D_{n}\left(s_{n}\right)\right)$;

(2): for all $i \in N, \vdash_{E_{i} F_{i}} \mathrm{~B}_{i}\left(\Gamma_{i}\right) \rightarrow \mathrm{B}_{i}\left(D_{i}\left(s_{i}\right)\right)$ for some $s_{i} \in S_{i}$,

where $E_{i}=\left\{\left(i_{1}, \ldots, i_{m}\right) \in E: i_{1}=i\right\} \cup\{\epsilon\}$ and $F_{i}=\left\{\left(i_{1}, \ldots, i_{m}\right) \in F: i_{1}=\right.$ $i\} \cup\{\epsilon\}$.

Since the disjunction before $\mathrm{B}_{i}\left(D_{i}\left(s_{i}\right)\right)$ is dropped in (2), statement (2) asserts the derivability of a particular decision and is more specific than (4.6). Also, this

\footnotetext{
${ }^{5}$ We may restrict the set of players into a subset of $N$. That is, some players reach decisions but the others do not. Especially, there are various possibilities for the latter players. In this paper, we do not go deep into this problem.
} 
becomes the form of (4.4) and enables us to look into player $i$ 's thought. This problem is the subject of Section 8 .

In the following, we focus on the provability of thought sequents of the form (4.6) rather than (4.7).

\subsection{Examples of admissible proofs and minimal inferential epistemic structures}

In the definition of a proof in $\mathrm{GL}_{E F}, F$ appears in P5, while $E$ appears in P1. That is, the outer $\mathrm{B}_{e}[\cdots]$ in a proof $P$ is constrained by $F$, and the entire description of the proof $P$ is constrained by $E$. For example, consider the thought sequent $\mathrm{B}_{1}\left(\Gamma_{1}\right) \rightarrow \mathrm{B}_{1}\left(d_{1}\left(\mathbf{s}_{11}\right)\right)=\mathrm{B}_{\epsilon}\left[\mathrm{B}_{1}\left(\Gamma_{1}\right) \rightarrow \mathrm{B}_{1}\left(d_{1}\left(\mathbf{s}_{11}\right)\right)\right]$, where $d_{1}\left(\mathbf{s}_{11}\right) \in \Gamma_{1}$. Here, player 1 has his belief set $\Gamma_{1}$ including $d_{1}\left(\mathbf{s}_{11}\right)$, i.e., 1 has the basic belief that his first strategy is a default decision. One possible proof for the sequent $\mathrm{B}_{1}\left(\Gamma_{1}\right) \rightarrow$ $\mathrm{B}_{1}\left(d_{1}\left(\mathbf{s}_{11}\right)\right)$ is as follows:

$$
\frac{\mathrm{B}_{1}\left[d_{1}\left(\mathbf{s}_{11}\right) \rightarrow d_{1}\left(\mathbf{s}_{11}\right)\right]}{\frac{\mathrm{B}_{\epsilon}\left[\mathrm{B}_{1}\left(d_{1}\left(\mathbf{s}_{11}\right)\right) \rightarrow \mathrm{B}_{1}\left(d_{1}\left(\mathbf{s}_{11}\right)\right)\right]}{\mathrm{B}_{\epsilon}\left[\mathrm{B}_{1}\left(\Gamma_{1}\right) \rightarrow \mathrm{B}_{1}\left(d_{1}\left(\mathbf{s}_{11}\right)\right)\right]}(\mathrm{Th})}\left(\mathrm{B}_{1} \rightarrow \mathrm{B}_{1}\right)
$$

This is an admissible proof when $E=F=\{\epsilon,(1)\}$, but is not when $E=\{\epsilon,(1)\}$ and $F=\{\epsilon\}$. However, $\mathrm{B}_{\epsilon}\left[\mathrm{B}_{1}\left(d_{1}\left(\mathbf{s}_{11}\right)\right) \rightarrow \mathrm{B}_{1}\left(d_{1}\left(\mathbf{s}_{11}\right)\right)\right]$ is an instance of the axiom in $\mathrm{GL}_{\{\epsilon,(1)\}\{\epsilon\}}$. This implies that

$$
\frac{\mathrm{B}_{\epsilon}\left[\mathrm{B}_{1}\left(d_{1}\left(\mathbf{s}_{11}\right)\right) \rightarrow \mathrm{B}_{1}\left(d_{1}\left(\mathbf{s}_{11}\right)\right)\right]}{\mathrm{B}_{\epsilon}\left[\mathrm{B}_{1}\left(\Gamma_{1}\right) \rightarrow \mathrm{B}_{1}\left(d_{1}\left(\mathbf{s}_{11}\right)\right)\right]}(\mathrm{Th})
$$

is an admissible proof in $\mathrm{GL}_{\{\epsilon,(1)\}\{\epsilon\}}$. Thus, $\mathrm{B}_{1}\left(\Gamma_{1}\right) \rightarrow \mathrm{B}_{1}\left(d_{1}\left(\mathbf{s}_{11}\right)\right)$ is provable both in $\mathrm{GL}_{\{\epsilon,(1)\}\{\epsilon,(1)\}}$ and $\mathrm{GL}_{\{\epsilon,(1)\}\{\epsilon\}}$.

The above examples state that the same sequent is obtained by proofs with different interpersonal epistemic depths. The proof of (4.8) has the redundancy in the investigator's thought about player 1's thought, while that of (4.9) has no redundancy in that sense. This inferential epistemic structure $F=\{\epsilon\}$ gives important information for the sequent $\mathrm{B}_{1}\left(\Gamma_{1}\right) \rightarrow \mathrm{B}_{1}\left(d_{1}\left(\mathbf{s}_{11}\right)\right)$. E.g., the above implies that any inferential structure $F$ (as well as $E \supseteq F$ ) works for the sequent $\mathrm{B}_{1}\left(\Gamma_{1}\right) \rightarrow \mathrm{B}_{1}\left(d_{1}\left(\mathbf{s}_{11}\right)\right)$. In general, it may be the case that a sequent has very complex descriptive epistemic structure $E$ but it requires only a small inferential epistemic structure $F$. To reflect this difference, we consider a minimal inferential epistemic structure $F$.

Let a thought sequent $\Gamma \rightarrow \Theta$ be given. We say that $F$ is minimal for $\Gamma \rightarrow \Theta$ iff $\vdash_{E F} \Gamma \rightarrow \Theta$ and $\nvdash_{E F^{\prime}} \Gamma \rightarrow \Theta$ for any epistemic structure $F^{\prime} \varsubsetneqq F$. Consider the above sequent $\mathrm{B}_{1}\left(\Gamma_{1}\right) \rightarrow \mathrm{B}_{1}\left(d_{1}\left(\mathbf{s}_{11}\right)\right)$ : Inferential epistemic structure $F=\{\epsilon\}$ is minimal for $\mathrm{B}_{1}\left(\Gamma_{1}\right) \rightarrow \mathrm{B}_{1}\left(d_{1}\left(\mathbf{s}_{11}\right)\right)$. Thus, the derivation of a default decision from the assumption of the same does not need the logical reasoning of player 1 . Nevertheless, player 1 is conscious of the pure default decision, which requires $E$ to contain (1). This is the argument on the default decision making mentioned in Section 1. 
The proof given in (4.1) holds for $i=1,2$. Abbreviating the outer $\mathrm{B}_{\epsilon}[\cdots]$, we have

$$
\vdash_{E_{i} F_{i}} \mathrm{~B}_{i}\left(\hat{g}_{i}^{1}\right) \rightarrow \mathrm{B}_{i}\left(\operatorname{Dom}_{i}\left(\mathbf{s}_{i 2}\right)\right),
$$

where $E_{i}=F_{i}=\{\epsilon,(i)\}$ for $i=1,2$. In fact, we can prove that this $F_{i}$ is a unique minimal inferential epistemic structure for $\mathrm{B}_{i}\left(\hat{g}_{i}^{1}\right) \rightarrow \mathrm{B}_{i}\left(\operatorname{Dom}_{i}\left(\mathbf{s}_{i 2}\right)\right)$. Similarly, we have, for $i=1,2$,

$$
\vdash_{E_{i} F_{i}} \mathrm{~B}_{i}\left(\hat{g}_{i}^{1}\right) \rightarrow \neg \mathrm{B}_{i}\left(\operatorname{Dom}_{i}\left(\mathbf{s}_{i 1}\right)\right) .
$$

In fact, this needs a slightly more complicated proof than (4.10). Indirectly, this provability itself can be seen as follows: In a similar manner as (4.1), we have $\vdash_{E_{i} F_{i}} \mathrm{~B}_{i}\left(\hat{g}_{i}^{1}\right) \rightarrow \mathrm{B}_{i}\left(\neg \operatorname{Dom}_{i}\left(\mathbf{s}_{i 1}\right)\right)$. Then (4.11) follows from this and the fact that $\vdash_{E_{i} F_{i}} \mathrm{~B}_{i}(\neg A) \rightarrow \neg \mathrm{B}_{i}(A)$ for any formula $A$ with $\delta^{r}\left(\mathrm{~B}_{i}(A)\right) \subseteq E_{i}$. This follows:

$$
\frac{\frac{\mathrm{B}_{i}[A \rightarrow A]}{\mathrm{B}_{i}[\neg A, A \rightarrow]}(\neg \rightarrow)}{\frac{\mathrm{B}_{i}(\neg A), \mathrm{B}_{i}(A) \rightarrow}{\mathrm{B}_{i}(\neg A) \rightarrow \neg \mathrm{B}_{i}(A)}(\rightarrow \neg)}\left(\mathrm{B}_{i} \rightarrow \mathrm{B}_{i}\right) .
$$

We give one more example of a provable sequent. Let $E_{2}=\{\epsilon,(2),(2,1)\}$ and $F_{2}=\{\epsilon,(2)\}$. From (4.10), we have, by (Th),

$$
\vdash_{E_{2} F_{2}} \mathrm{~B}_{2}\left(\hat{g}_{2}^{1}\right), \mathrm{B}_{2} \mathrm{~B}_{1}\left(\hat{g}_{1}^{1}\right) \rightarrow \mathrm{B}_{2}\left(\operatorname{Dom}_{2}\left(\mathbf{s}_{22}\right)\right) .
$$

This $E_{2}$ is needed for this sequent, while $F_{2}=\{\epsilon,(2)\}$ is a unique minimal inferential epistemic structure.

Now, consider the decision criterion $\mathscr{D}_{2}^{2}$ for player 2 . Similar to the derivation of (4.10), we have

$$
\frac{\frac{\mathrm{B}_{2} \mathrm{~B}_{1}\left[\hat{g}_{1}^{2} \rightarrow \mathrm{Dom}_{1}\left(\mathbf{s}_{12}\right)\right]}{\mathrm{B}_{2}\left[\mathrm{~B}_{1}\left(\hat{g}_{1}^{2}\right) \rightarrow \mathrm{B}_{1}\left(\operatorname{Dom}_{1}\left(\mathbf{s}_{12}\right)\right)\right]}\left(\mathrm{B}_{1} \rightarrow \mathrm{B}_{1}\right)}{\frac{\mathrm{B}_{2}\left[\mathrm{~B}_{1}\left(\hat{g}_{1}^{2}\right) \rightarrow \bigvee_{s_{1}} \mathrm{~B}_{1}\left(\operatorname{Dom}_{1}\left(s_{1}\right)\right)\right]}{\mathrm{B}_{2} \mathrm{~B}_{1}\left(\hat{g}_{1}^{2}\right) \rightarrow \mathrm{B}_{2}\left(\bigvee_{s_{1}} \mathrm{~B}_{1}\left(\operatorname{Dom}_{1}\left(s_{1}\right)\right)\right)}\left(\mathrm{B}_{2} \rightarrow \mathrm{B}_{2}\right)}(\rightarrow \bigvee)
$$

Thus, for $E_{2}=F_{2}=\{\epsilon,(2),(2,1)\}$,

$$
\vdash_{E_{2} F_{2}} \mathrm{~B}_{2} \mathrm{~B}_{1}\left(\hat{g}_{1}^{2}\right) \rightarrow \mathrm{B}_{2}\left(\bigvee_{s_{1}} \mathrm{~B}_{1}\left(\operatorname{Dom}_{1}\left(s_{1}\right)\right)\right) .
$$

That is, player 2 predicts that 1 can choose some decision following DC1. In addition, 2 can predict what 1 would choose. That is, the following hold: $\vdash_{E_{2} F_{2}}$ $\mathrm{B}_{2} \mathrm{~B}_{1}\left(\hat{g}_{1}^{2}\right) \rightarrow \mathrm{B}_{2} \mathrm{~B}_{1}\left(D_{1}^{1}\left(\mathbf{s}_{12}\right)\right)$ and $\vdash_{E_{2} F_{2}} \mathrm{~B}_{2} \mathrm{~B}_{1}\left(\hat{g}_{1}^{2}\right) \rightarrow \mathrm{B}_{2}\left(\neg \mathrm{B}_{1}\left(D_{1}^{1}\left(\mathbf{s}_{11}\right)\right)\right)$. It follows from these that

$$
\vdash_{E_{2} F_{2}} \mathrm{~B}_{2}\left(\hat{g}_{2}^{2}\right), \mathrm{B}_{2} \mathrm{~B}_{1}\left(\hat{g}_{1}^{2}\right) \rightarrow \mathrm{B}_{2}\left(\bigwedge_{s_{1}}\left(\mathrm{~B}_{1}\left(\operatorname{Dom}_{1}\left(s_{1}\right)\right) \supset \operatorname{Best}_{2}\left(\mathbf{s}_{22} \mid s_{1}\right)\right)\right) .
$$


That is, $\mathbf{S}_{22}$ is a best strategy to whatever 1 would choose. Combining (4.13) and (4.14), we obtain

$$
\vdash_{E_{2} F_{2}} \mathrm{~B}_{2}\left(\hat{g}_{2}^{2}\right), \mathrm{B}_{2} \mathrm{~B}_{1}\left(\hat{g}_{1}^{2}\right) \rightarrow \mathrm{B}_{2}\left(D_{2}^{2}\left(\mathbf{s}_{22}\right)\right) .
$$

Hence, player 2 derives his decision $\mathbf{S}_{22}$ satisfying decision criterion DC2 from $\mathrm{B}_{2} \mathrm{~B}_{1}\left(\hat{g}_{1}^{2}\right) \cup \mathrm{B}_{2}\left(\hat{g}_{2}^{2}\right)$. For this, $E_{2}$ and $F_{2}$ must be $\{\epsilon,(2),(2,1)\}$.

Finally, consider the default decision criterion $\mathscr{D}_{i}^{0}$. For example, suppose that player 1 has the beliefs on the game $g^{1}$ of Table 1 and that he has the belief that his default decision is $\mathbf{s}_{11}$ and not $\mathbf{s}_{12}$. In this case, the question is what $E$ and $F$ are required in order to prove the thought sequent

$$
\mathrm{B}_{1}\left(\hat{g}^{1}\right), \mathrm{B}_{1}\left(d_{1}\left(\mathbf{s}_{11}\right)\right), \mathrm{B}_{1}\left(\neg d_{1}\left(\mathbf{s}_{12}\right)\right) \rightarrow \mathrm{B}_{1}\left(d_{1}\left(\mathbf{s}_{11}\right)\right) .
$$

Since $\delta(A)=\{(1)\}$ for each $A$ in this sequent, the minimum $E$ must be $\{\epsilon,(1)\}$. On the other hand, the thought sequent of (4.16) is a special case of the endsequent of (4.8). Thus the minimal inferential structure $F$ for the sequent of (4.16) is $\{\epsilon\}$.

To discuss the minimality of an inferential structure and its game theoretical applications, we need various meta-theorems, which will be given in Section 6 .

\subsection{Cut-elimination theorem}

The relation between the sequent formulation of $\mathrm{KD}^{n}$ and the above $\mathrm{GL}_{E F}$ is as follows. The sequent formulation of $\mathrm{KD}^{n}$ is obtained by imposing no restrictions on admissible formulae and on admissible proofs. Specifically, we delete all the outer $\mathrm{B}_{e}[\cdots]$ from all the thought sequents in the axiom and inference rules, where all formulae are taken simply from $\mathscr{P}$. This sequent calculus $\mathrm{KD}^{n}$ is standard in the logic literature, which corresponds to the sequent form of KD $4^{n}$ briefly mentioned in Kaneko [4].

We have a more accurate relationship to $\mathrm{KD}^{n}$, which is due to Kaneko and Suzuki [7].

Theorem 4.2 (Relation to $\mathbf{K D}^{n}$ ). Let $\Gamma$ and $\Theta$ be finite sets of formulae, and let $E$ be any epistemic structure with $\delta(\Gamma \cup \Theta) \subseteq E$. Then $\vdash \operatorname{KD}^{n} \Gamma \rightarrow \Theta$ if and only if $\vdash_{E E} \Gamma \rightarrow \Theta$.

The following theorem is the key-theorem for $\mathrm{GL}_{E F}$.

Theorem 4.3 (Cut-Elimination). If $\vdash_{E F} \mathrm{~B}_{e}[\Gamma \rightarrow \Theta]$, then there is a cut-free proof $P$ of $\mathrm{B}_{e}[\Gamma \rightarrow \Theta]$ in $\mathrm{GL}_{E F}$.

This is also mentioned in Kaneko and Suzuki [7]. A proof of this theorem is obtained by modifying and simplifying the proof of the cut-elimination theorem for the infinitary predicate logic $\mathrm{GL}_{m}$ in Kaneko and Nagashima [6]. Theorem 4.3 will be one of the key theorems to investigate interpersonal epistemic inferential complexities, in particular, minimalities of inferential epistemic structures. 


\section{Prediction-decision criteria}

In this section, we give a general definition of a prediction-decision criterion, and also look at how the previous prediction-decision criteria are formulated within our general definition.

A prediction-decision criterion $\mathscr{D}_{i}=\left\{D_{i}\left(s_{i}\right): s_{i} \in S_{i}\right\}$ for player $i$ is defined based on a prediction criterion $\mathscr{T}_{i}$ and a valuation criterion $\mathscr{R}_{i}$ of actions. These are given as follows:

(1): $\mathscr{P} i=\left\{\operatorname{Pre}_{i j}\left(s_{j}\right): s_{j} \in S_{j}\right.$ and $\left.j \in N-\{i\}\right\}$, where each $\operatorname{Pre}_{i j}\left(s_{j}\right)$ is a formula indexed by $i, j(j \neq i)$ and $s_{j} \in S_{j}$.

(2): $\mathscr{R}_{i}=\left\{\operatorname{Res}_{i}\left(s_{i} \mid s_{-i}\right): s_{i} \in S_{i}\right.$ and $\left.s_{-i} \in S_{-i}\right\}$, where each $\operatorname{Res}_{i}\left(s_{i} \mid s_{-i}\right)$ is a formula indexed by $i, s_{i} \in S_{i}$ and $s_{-i} \in S_{-i}$.

The first one, $\mathscr{P}_{i}$, describes player $i$ 's prediction about other players' choices, and the second one, $\mathscr{R}_{i}$, does the valuations of responses to predicted strategies. In the following, we denote $\bigwedge_{j \neq i} \operatorname{Pre}_{i j}\left(s_{j}\right)$ by $\operatorname{Pre}_{i}\left(s_{-i}\right)$, which means that $s_{-i}$ is an $(n-1)$-tuple of predicted strategies by player $i$. Based on $\mathscr{P}_{i}$ and $\mathscr{R}_{i}$, the decision criterion $\mathscr{D}_{i}=\left\{D_{i}\left(s_{i}\right): s_{i} \in S_{i}\right\}$ is defined as follows: for $s_{i} \in S_{i}$,

$$
D_{i}\left(s_{i}\right)=\bigvee_{s_{-i}} \operatorname{Pre}_{i}\left(s_{-i}\right) \wedge \bigwedge_{s_{-i}}\left(\operatorname{Pre}_{i}\left(s_{-i}\right) \supset \operatorname{Res}_{i}\left(s_{i} \mid s_{-i}\right)\right) .
$$

Here, $\bigvee_{s_{-i}}$ and $\bigwedge_{s_{-i}}$ are abbreviations of $\bigvee_{s_{-i} \in S_{-i}}$ and $\bigwedge_{s_{-i} \in S_{-i}}$. The definition (5.1) states that player $i$ has a prediction about what the other players would choose and his decision is an appropriate response to the predicted strategies.

Now, we look at how (5.1) captures the examples of prediction-decision criteria discussed earlier. Those examples except the last two are for 2-person games. In the following, let $P_{i j}^{0}\left(s_{j}\right)=\top$ for $s_{j} \in S_{j}(j \neq i)$.

DC1: Consider $\mathscr{D}_{i}^{1}=\left\{\operatorname{Dom}_{i}\left(s_{i}\right): s_{i} \in S_{i}\right\} . \operatorname{Dom}_{i}\left(s_{i}\right)$ is equivalent to, in classical logic (i.e., $\mathrm{GL}_{\{\epsilon\}\{\epsilon\}}$ ),

$$
\bigvee_{s_{j}} P_{i j}^{0}\left(s_{j}\right) \wedge \bigwedge_{s_{j}}\left(P_{i j}^{0}\left(s_{j}\right) \supset \operatorname{Best}_{i}\left(s_{i} \mid s_{j}\right)\right) .
$$

Note that $s_{-i}$ is the same as $s_{j}$ because $N=\{1,2\}$. Hence, $\mathscr{D}_{i}^{1}$ is regarded as a special case of (5.1).

DC2: This is given by (3.3) as $\mathscr{D}_{i}^{2}=\left\{\bigvee_{s_{j}} \mathrm{~B}_{j}\left(\operatorname{Dom}_{j}\left(s_{j}\right)\right) \wedge \bigwedge_{s_{j}}\left(\mathrm{~B}_{j}\left(\operatorname{Dom}_{j}\left(s_{j}\right)\right) \supset\right.\right.$ $\left.\left.\operatorname{Best}_{i}\left(s_{i} \mid s_{j}\right)\right): s_{i} \in S_{i}\right\}$. In this case, $\mathscr{P}_{i}^{2}=\left\{\mathrm{B}_{j}\left(\operatorname{Dom}_{j}\left(s_{j}\right)\right): s_{j} \in S_{j}\right\}$ and $\mathscr{R}_{i}^{2}=\left\{\operatorname{Best}_{i}\left(s_{i} \mid s_{j}\right): s_{i} \in S_{i}\right.$ and $\left.s_{j} \in S_{j}\right\}(j \neq i)$.

DC0: The pure default decision criterion $\mathscr{D}_{i}^{0}=\left\{d_{i}\left(s_{i}\right): s_{i} \in S_{i}\right\}$ can be formulated as $\bigvee_{s_{j}} P_{i j}^{0}\left(s_{j}\right) \wedge \bigwedge_{s_{j}}\left(P_{i j}^{0}\left(s_{j}\right) \supset d_{i}\left(s_{i}\right)\right)$ for $s_{i} \in S_{i}$.

DC20: The dogmatic prediction decision criterion is given as $\mathscr{D}_{i}^{20}=$ $\left\{\bigvee_{s_{j}} \mathrm{~B}_{j}\left(d_{j}\left(s_{j}\right)\right) \wedge \bigwedge_{s_{j}}\left(\mathrm{~B}_{j}\left(d_{j}\left(s_{j}\right)\right) \supset \operatorname{Best}_{i}\left(s_{i} \mid s_{j}\right)\right): s_{i} \in S_{i}\right\}$. In this case, the 
prediction criterion is given as $\mathscr{P}_{i}^{20}=\left\{\mathrm{B}_{j}\left(d_{j}\left(s_{j}\right)\right): s_{j} \in S_{j}\right\}(j \neq i)$, and the valuation criterion is $\mathscr{R}_{i}^{2}$. To make this criterion effective, player $i$ needs some beliefs about player $j$ 's default decision.

$\mathbf{v N ( M a x i m i n ) : ~ I n ~ t h e ~ n o n - f o r m a l i z e d ~ l a n g u a g e , ~ t h e ~ m a x i m i n ~ d e c i s i o n ~ c r i t e r i o n ~}$ for $i=1$ is described as a strategy $s_{1}$ maximizing $\min _{s_{2}} g_{1}\left(s_{1}, s_{2}\right)$. This is equivalent to that for any $s_{2} \in S_{2}$ and $t_{1} \in S_{1}$, there is another $t_{2} \in S_{2}$ such that $P_{1}\left(s_{1}, s_{2}: t_{1}, t_{2}\right)$ holds. This is described as the following formula:

$$
\bigwedge_{s_{2}} \bigwedge_{t_{1}} \bigvee_{t_{2}} P_{1}\left(s_{1}, s_{2}: t_{1}, t_{2}\right)
$$

Hence the maximin decision criterion is given as $\mathscr{D}_{1}^{v N}=\left\{D_{1}^{v N}\left(s_{1}\right): s_{1} \in S_{1}\right\}=$ $\left\{\bigwedge_{s_{2}} \bigwedge_{t_{1}} \bigvee_{t_{2}} P_{1}\left(s_{1}, s_{2}: t_{1}, t_{2}\right): s_{1} \in S_{1}\right\}$. Each $D_{1}^{v N}\left(s_{1}\right)$ is further equivalent to $\bigvee_{s_{2}} P_{12}^{0}\left(s_{2}\right) \wedge \bigwedge_{s_{2}}\left(P_{12}^{0}\left(s_{2}\right) \supset \bigwedge_{t_{1}} \bigvee_{t_{2}} P_{1}\left(s_{1}, s_{2}: t_{1}, t_{2}\right)\right)$, which is a special case of (5.1). The criterion for player 2 is formulated in a symmetric manner.

Consider the hierarchical decision criterion HDC and negative prediction criterion NPC in the 3-person case:

HDC: We define the prediction criterion $\mathscr{P}_{1}^{H}=\left\{\operatorname{Pre}_{1 j}^{H}\left(s_{j}\right): s_{j} \in S_{j}\right.$ and $\left.j=2,3\right\}$ of player 1 as follows:

(1): $\operatorname{Pre}_{12}^{H}\left(s_{2}\right)=B_{2}\left(\bigvee_{s_{-2}}\left(P_{21}^{0}\left(s_{1}\right) \wedge \mathrm{B}_{3}\left(\operatorname{Dom}_{3}\left(s_{3}\right)\right)\right) \wedge\right.$

$$
\left.\bigwedge_{s_{-2}}\left(P_{21}^{0}\left(s_{1}\right) \wedge \mathrm{B}_{3}\left(\operatorname{Dom}_{3}\left(s_{3}\right)\right) \supset \operatorname{Best}_{2}\left(s_{2} \mid s_{-2}\right)\right)\right) \text { for all } s_{2} \in S_{2}
$$

(2): $\operatorname{Pre}_{13}^{H}\left(s_{3}\right)=P_{13}^{0}\left(s_{3}\right)=\top$ for all $s_{3} \in S_{3}$, i.e., 1 does not predict 3's choice.

Now, $\mathscr{D}_{1}^{H}=\left\{D_{1}^{H}\left(s_{1}\right): s_{1} \in S_{1}\right\}$ is given as

(3): $\bigvee_{s_{-1}} \operatorname{Pre}_{1}^{H}\left(s_{-1}\right) \wedge \bigwedge_{s_{-1}}\left(\operatorname{Pre}_{1}^{H}\left(s_{-1}\right) \supset \operatorname{Best}_{1}\left(s_{1} \mid s_{-1}\right)\right)$,

where $\operatorname{Pre}_{1}^{H}\left(s_{-1}\right)=\operatorname{Pre}_{12}^{H}\left(s_{2}\right) \wedge \operatorname{Pre}_{13}^{H}\left(s_{3}\right)$. Thus, HDC is a special case of (5.1). Notice that $\operatorname{Pre}_{12}^{H}(\cdot)$ is also taking the form of (5.1). That is, we would find a structure parallel to (5.1) in the prediction criterion. This parallelism will be discussed in Section 8.

NPC: We define the prediction criterion $\mathscr{P}_{1}^{N}=\left\{\operatorname{Pre}_{1 j}\left(s_{j}\right): s_{j} \in S_{j}\right.$ and $\left.j=2,3\right\}$ as

(1): $\operatorname{Pre}_{1 j}^{N}\left(s_{j}\right)=\mathrm{B}_{j}\left(\operatorname{Und}_{j}\left(s_{j}\right)\right)$ for $s_{j} \in S_{j}$ and $j \neq 1$

That is, player 1 predicts that player $j$ would play a undominated strategy. Then each formula in $\mathscr{D}_{1}^{N}=\left\{D_{1}^{N}\left(s_{1}\right): s_{1} \in S_{1}\right\}$ is given as

(2): $\bigvee_{s_{-1}} \operatorname{Pre}_{1}^{N}\left(s_{-1}\right) \wedge \bigwedge_{s_{-1}}\left(\operatorname{Pre}_{1}^{N}\left(s_{-1}\right) \supset \operatorname{Best}_{1}\left(s_{1} \mid s_{-1}\right)\right)$.

\section{Various meta-theorems}

In this section, we provide various meta-theorems to be used in the logico-game analysis of decision making. The reader who is interested only in the game 
theoretical results can skip this section. The first two theorems are proved in Kaneko and Suzuki [8] in the model-theoretic manner, and the others are proved in Kaneko and Suzuki [9] in the proof-theoretic manner.

Let $S$ be a subset of $N \cup\{0\}$, and $A$ a formula in $\mathscr{P}$. We say that $A$ is an $S$-formula iff

(i) $e=\left(i_{1}, \ldots, i_{m}\right) \in \delta^{r}(A)$ and $m \geq 1$ imply $i_{1} \in S$;

(ii) $\epsilon \in \delta^{r}(A)$ implies $0 \in S$.

For example, $\mathrm{B}_{2} \mathrm{~B}_{1}\left(\operatorname{Dom}_{1}\left(s_{1}\right)\right)$ is a $\{2\}$-formula. Any $\{0\}$-formula is nonepistemic.

We have the following separation theorem.

Theorem 6.1 (Epistemic separation). Let $S_{1}, \ldots, S_{k}$ be disjoint nonempty subsets of $N \cup\{0\}$. Let $\Gamma_{t}$ be a finite set of $S_{t}$-formulae and $A_{t}$ an $S_{t}$-formula for $t=1, \ldots, k$. Also, we let $E_{t}=\left\{\left(i_{1}, \ldots, i_{m}\right) \in E: i_{1} \in S_{t}\right\} \cup\{\epsilon\}$ and $F_{t}=$ $\left\{\left(i_{1}, \ldots, i_{m}\right) \in F: i_{1} \in S_{t}\right\} \cup\{\epsilon\}$ for $t=1, \ldots, k$.

(1): Suppose that $\Gamma_{t}$ is consistent for $t=1, \ldots, k$. Then $\vdash_{E F} \Gamma_{1}, \ldots, \Gamma_{k} \rightarrow A_{1} \wedge$ $\ldots \wedge A_{k}$ if and only if $\vdash_{E_{t} F_{t}} \Gamma_{t} \rightarrow A_{t}$ for all $t=1, \ldots, k$.

(2): $\vdash_{E F} \Gamma_{1}, \ldots, \Gamma_{k} \rightarrow A_{1} \vee \ldots \vee A_{k}$ if and only if $\vdash_{E_{t} F_{t}} \Gamma_{t} \rightarrow A_{t}$ for some $t=1, \ldots, k$.

We need to evaluate the provability of sequents such as $\mathrm{B}_{i}\left(\Gamma_{i}\right) \rightarrow$ $\bigvee_{s_{i}} \mathrm{~B}_{i}\left(D_{i}\left(s_{i}\right)\right)$ of (4.6). For this purpose, the next theorem is provided.

Theorem 6.2 (Epistemic disjunction). Let $\Gamma$ be a finite set of formulae and $\Phi$ a finite nonempty set of formulae. Then $\vdash_{E F} \mathrm{~B}_{i}\left(\Gamma_{i}\right) \rightarrow \bigvee \mathrm{B}_{i}(\Phi)$ if and only if $\vdash_{E F}$ $\mathrm{B}_{i}\left(\Gamma_{i}\right) \rightarrow \mathrm{B}_{i}(A)$ for some $A \in \Phi$.

The if part is proved with $(\rightarrow \bigvee)$. The only-if part is essential here. It can be proved using the cut-elimination theorem. However, Kaneko and Suzuki [8] proved Theorem 6.2 using model-theoretic surgical operations.

Using Theorems 6.1 and 6.2, we can prove Theorem 4.1.

Proof of Theorem 4.1. The derivation of (1) from (2) is straightforward. Conversely, suppose (1). Then $\vdash_{E F} \Gamma_{0}, \mathrm{~B}_{1}\left(\Gamma_{1}\right), \ldots, \mathrm{B}_{n}\left(\Gamma_{n}\right) \rightarrow \top \wedge\left(\bigvee_{s_{1}} \mathrm{~B}_{1}\left(D_{1}\left(s_{1}\right)\right)\right.$ $\wedge \cdots \wedge\left(\bigvee_{s_{n}} \mathrm{~B}_{n}\left(D\left(s_{n}\right)\right)\right)$. By Theorem 6.1.(1), we have $\vdash_{E_{i} F_{i}} \mathrm{~B}_{i}\left(\Gamma_{i}\right) \rightarrow$ $\bigvee_{s_{i}} \mathrm{~B}_{i}\left(D_{i}\left(s_{i}\right)\right)$ for each $i \in N$. Then, by Theorem 6.2, we have (2).

Here, we give another theorem and two more lemmas, which will be used for evaluations of an inferential epistemic structure $F$ for a given sequent. Proofs of them are given in Kaneko and Suzuki [9].

Theorem 6.3 (Epistemic inferences). Let $\Gamma$ be a finite set of formulae and $A$ a formula.

(1): If $\vdash_{E F} \mathrm{~B}_{i}(\Gamma) \rightarrow$ or $\vdash_{E F} \rightarrow \mathrm{B}_{i}(A)$, then $(i) \in F$.

(2): If $\vdash_{E F} \mathrm{~B}_{i}(\Gamma) \rightarrow \mathrm{B}_{i}(A)$ and $A \notin \Gamma$, then $(i) \in F$. 
(3): Let $F$ be a minimal inferential epistemic structure for $\mathrm{B}_{i}(\Gamma) \rightarrow \mathrm{B}_{i}(A)$. Then $A \in \Gamma$ implies $F=\{\epsilon\}$.

(4): If $F=\{\epsilon\}$ and $\vdash_{E F} \mathrm{~B}_{i}(\Gamma) \rightarrow \mathrm{B}_{i}(A)$, then $A \in \Gamma$.

To exemplify how this theorem and the next two lemmas are used, we consider the thought sequent $\rightarrow \mathrm{B}_{2} \mathrm{~B}_{1}(p \supset p)$, where $p \in A F$. It holds that $\vdash_{E F}$ $\rightarrow \mathrm{B}_{2} \mathrm{~B}_{1}(p \supset p)$, where $E=F=\{\epsilon,(2),(2,1)\}$. Let us see the minimality of $F=\{\epsilon,(2),(2,1)\}$ for $\rightarrow \mathrm{B}_{2} \mathrm{~B}_{1}(p \supset p)$. Applying Theorem 6.3.(1) to this thought sequent, we have $(2) \in F$. It remains to show $(2,1) \in F$. For this step, the following two lemmas are useful.

Lemma 6.4. Consider a thought sequent $\mathrm{B}_{i}(\Gamma) \rightarrow \mathrm{B}_{i}(\Theta)$ with $|\Theta| \leq 1$. If $\vdash_{E F}$ $\mathrm{B}_{i}(\Gamma) \rightarrow \mathrm{B}_{i}(\Theta)$ and $(i) \in F$, then $\vdash_{E F} \mathrm{~B}_{i}[\Gamma \rightarrow \Theta]$.

For an epistemic structure $E$ and $(i) \in E$, we write $E_{-i}=\{e: i \circ e \in E\}$. Then $E_{-i}$ is also an epistemic structure. For example, when $E=\{\epsilon,(2),(2,1)\}$, we have $E_{-2}=\{\epsilon,(1)\}$.

Lemma 6.5. Let $(i) \in F$. Then $\vdash_{E F} \mathrm{~B}_{i}[\Gamma \rightarrow \Theta]$ if and only if $\vdash_{E_{-i} F_{-i}} \Gamma \rightarrow \Theta$.

Let us return to the minimality of $F=\{\epsilon,(2),(2,1)\}$ for $\rightarrow \mathrm{B}_{2} \mathrm{~B}_{1}(p \supset p)$. By Lemma 6.4, we have $\vdash_{E F} \mathrm{~B}_{2}\left[\rightarrow \mathrm{B}_{1}(p \supset p)\right]$. By Lemma 6.5, we have $\vdash_{E_{-2} F_{-2}} \rightarrow \mathrm{B}_{1}(p \supset p)$. Again, by Theorem 6.3.(1), we have (1) $\in F_{-2}$, which implies $(2,1) \in F$.

\section{Minimal inferential epistemic structures}

Theorem 4.1 guarantees that we consider separately the provability of an individual statement:

$$
\vdash_{E_{i} F_{i}} \mathrm{~B}_{i}\left(\Gamma_{i}\right) \rightarrow \mathrm{B}_{i}\left(D_{i}\left(s_{i}\right)\right) .
$$

We evaluate a minimal $F_{i}$ for this sequent. In fact, Theorem 6.3 already gives some information about a required $F_{i}$. When $D_{i}\left(s_{i}\right) \notin \Gamma_{i}$, we have $(i) \in F_{i}$. Here, we consider the previous examples.

DC1: Recall (4.10), i.e., $\vdash_{E_{i} F_{i}} \mathrm{~B}_{i}\left(\hat{g}_{i}^{1}\right) \rightarrow \mathrm{B}_{i}\left(\operatorname{Dom}_{i}\left(\mathbf{s}_{i 2}\right)\right)$, where $E_{i}=F_{i}=\{\epsilon,(i)\}$. This $F_{i}$ is a unique minimal one for this sequent. Indeed, since $\operatorname{Dom}_{i}\left(\mathbf{s}_{i 2}\right) \notin \hat{g}_{i}^{1}$, we have $(i) \in F_{i}$ by Theorem 6.3.

DC2: Recall (4.15), i.e., $\vdash_{E_{2} F_{2}} \mathrm{~B}_{2}\left(\hat{g}_{2}^{2}\right), \mathrm{B}_{2} \mathrm{~B}_{1}\left(\hat{g}_{1}^{2}\right) \rightarrow \mathrm{B}_{2}\left(D_{2}^{2}\left(\mathbf{s}_{22}\right)\right)$, where $E_{2}=F_{2}=$ $\{\epsilon,(2),(2,1)\}$. In this case, $F_{2}$ is a unique minimal one. Here, we give a proof for this fact, which needs some steps.

Let $F_{2}$ be any epistemic structure for which (4.15) holds. By Theorem 6.3.(2), we have $(2) \in F_{2}$. It remains to show $(2,1) \in F_{2}$. Applying Lemma 6.4 to (4.15), we have

$$
\vdash_{E_{2} F_{2}} \mathrm{~B}_{2}\left[\hat{g}_{2}^{2}, \mathrm{~B}_{1}\left(\hat{g}_{1}^{2}\right) \rightarrow D_{2}^{2}\left(\mathbf{s}_{22}\right)\right],
$$

and then, by Lemma 6.5, 


$$
\vdash_{E_{-2} F_{-2}} \hat{g}_{2}^{2}, \mathrm{~B}_{1}\left(\hat{g}_{1}^{2}\right) \rightarrow D_{2}^{2}\left(\mathbf{s}_{22}\right),
$$

where $E_{-2}=\left\{e: 2 \circ e \in E_{2}\right\}$ and $F_{-2}=\left\{e: 2 \circ e \in F_{2}\right\}$. Since $D_{2}^{2}\left(\mathbf{s}_{22}\right)=$ $\bigvee_{s_{1}} \mathrm{~B}_{1}\left(\operatorname{Dom}_{1}\left(s_{1}\right)\right) \wedge \bigwedge_{s_{1}}\left(\mathrm{~B}_{1}\left(\operatorname{Dom}_{1}\left(s_{1}\right)\right) \supset \operatorname{Best}_{2}\left(\mathbf{s}_{22} \mid s_{1}\right)\right)$, we have

$$
\vdash_{E_{-2} F_{-2}} \hat{g}_{2}^{2}, \mathrm{~B}_{1}\left(\hat{g}_{1}^{2}\right) \rightarrow \bigvee_{s_{1}} \mathrm{~B}_{1}\left(\operatorname{Dom}_{1}\left(s_{1}\right)\right)
$$

This implies $\vdash_{E_{-2} F_{-2}} \hat{g}_{2}^{2}, \mathrm{~B}_{1}\left(\hat{g}_{1}^{2}\right) \rightarrow \perp \vee \bigvee_{s_{1}} \mathrm{~B}_{1}\left(\operatorname{Dom}_{1}\left(s_{1}\right)\right)$. By Theorem 6.1, we have $\vdash_{E_{-2} F_{-2}} \hat{g}_{2}^{2} \rightarrow \perp$ or $\vdash_{E_{-2} F_{-2}} \mathrm{~B}_{1}\left(\hat{g}_{1}^{2}\right) \rightarrow \bigvee_{s_{1}} \mathrm{~B}_{1}\left(\operatorname{Dom}_{1}\left(s_{1}\right)\right)$. Since $\hat{g}_{2}^{2}$ is consistent, the latter is the case. Then, by Theorem $6.2, \vdash_{E_{-2} F_{-2}} \mathrm{~B}_{1}\left(\hat{g}_{1}^{2}\right) \rightarrow$ $\mathrm{B}_{1}\left(\operatorname{Dom}_{1}\left(s_{1}\right)\right)$ for some $s_{1}$. Since $\operatorname{Dom}_{1}\left(s_{1}\right) \notin \hat{g}_{1}^{2}$, we have $(1) \in F_{-2}$, which implies $(2,1) \in F_{2}$.

Undecidability with DC2 and $F_{2}=\{\epsilon,(2)\}$ : If we assume $F_{2}=\{\epsilon,(2)\}$, then we can prove the following unprovability results:

(U1): $\nvdash_{E_{2} F_{2}} \mathrm{~B}_{2}\left(\hat{g}_{2}^{2}\right), \mathrm{B}_{2} \mathrm{~B}_{1}\left(\hat{g}_{1}^{2}\right) \rightarrow \mathrm{B}_{2}\left(D_{2}^{2}\left(\mathbf{s}_{22}\right)\right)$;

(U2): $\nvdash_{E_{2} F_{2}} \mathrm{~B}_{2}\left(\hat{g}_{2}^{2}\right), \mathrm{B}_{2} \mathrm{~B}_{1}\left(\hat{g}_{1}^{2}\right) \rightarrow \mathrm{B}_{2}\left(\neg D_{2}^{2}\left(\mathbf{s}_{22}\right)\right)$;

(U3): $\nvdash_{E_{2} F_{2}} \mathrm{~B}_{2}\left(\hat{g}_{2}^{2}\right), \mathrm{B}_{2} \mathrm{~B}_{1}\left(\hat{g}_{1}^{2}\right) \rightarrow \neg \mathrm{B}_{2}\left(D_{2}^{2}\left(\mathbf{s}_{22}\right)\right)$.

Fact $\mathrm{U} 1$ follows the minimality of $\{\epsilon,(2),(2,1)\}$ for the sequent. Facts $\mathrm{U} 2$ and U3 can be proved in various manners. ${ }^{6}$ These differ in the positions of the negation symbol $\neg$ : $\mathrm{U} 2$ states that player 2 himself cannot reach $\neg D_{2}^{2}\left(\mathbf{s}_{22}\right)$ (from the viewpoint of the investigator), but $\mathrm{U} 3$ that the investigator does not derive from $\mathrm{B}_{2}\left(\hat{g}_{2}^{2}\right), \mathrm{B}_{2} \mathrm{~B}_{1}\left(\hat{g}_{1}^{2}\right)$ that 2 does not believe $D_{2}^{2}\left(\mathbf{s}_{22}\right)$. By these three facts, we have the conclusion that player 2 cannot rationally decide whether or not he could reach a decision with criterion DC2 without reading player 1's mind. It will be shown in Section 8 that these unprovabilities can be stated from the viewpoint of player 2 .

The above undecidability results $\mathrm{U} 1, \mathrm{U} 2$ and $\mathrm{U} 3$ hold even if we change game $g^{2}$ to $g^{1}$ and keep criterion DC2 with $E_{2}=\{\epsilon,(2),(2,1)\}$ but $F_{2}=\{\epsilon,(2)\}$. In this case, however, if player 2 switches his criterion back to criterion DC1, then he could make a decision in $g^{1}$ without reading 1's mind.

The above considerations are paraphrased in terms of inferential complexities. In game $g^{1}$, decision criterion DC1 gives a decision, but in game $g^{2}$, it is incapable of giving a decision to player 2. A remedy for this incapability is to change DC1 to DC2 including the prediction over player 1's decision. However, this remedy requires a deeper inferential complexity of interpersonal introspections, i.e., player 2 need to read player 1's mind.

\footnotetext{
${ }^{6}$ One proof of $\mathrm{U} 2$ is as follows: Let $\varepsilon$ be the B-eliminating operator $\varepsilon$, i.e., $\varepsilon A$ is obtained from formula $A$ by eliminating all occurrences of $\mathrm{B}_{1}, \ldots, \mathrm{B}_{n}$ in $A$. Then it can be proved that if $\vdash_{E F} \Gamma \rightarrow \Theta$, then $\vdash_{\{\epsilon\}\{\epsilon\}} \varepsilon \Gamma \rightarrow \varepsilon \Theta$. Now, suppose the sequent of $\mathrm{U} 2$ is provable. We apply the above result to the sequent of $\mathrm{U} 2$, and obtain $\vdash_{\{\epsilon\}\{\epsilon\}} \hat{g}_{2}^{2}, \hat{g}_{1}^{2} \rightarrow \neg D_{2}^{2}\left(\mathbf{s}_{22}\right)$. This is impossible. U3 can be proved in the same manner.
} 
Although we do not repeat paraphrastic interpretations like the above paragraph, it would be helpful to think about the arguments in the subsequent sections with the above paraphrastic manners.

Let us see minimal inferential epistemic structures in situations with other prediction-decision criteria.

DC0: Default decision criterion $\mathscr{D}_{1}^{0}$ is derived from a dogmatic belief on default decisions. Suppose $d_{1}\left(\mathbf{s}_{11}\right) \in \Gamma_{1}$. Then it is proved in (4.8) that $\vdash_{E_{1} F_{1}} \mathrm{~B}_{1}\left(\Gamma_{1}\right) \rightarrow$ $\mathrm{B}_{1}\left(d_{1}\left(\mathbf{s}_{11}\right)\right)$. By $d_{1}\left(\mathbf{s}_{11}\right) \in \Gamma_{1}$ and Theorem 6.3.(2), $F_{1}=\{\epsilon\}$ is a unique minimal inferential epistemic structure. There is no subtlety in criterion DC0. However, we meet some subtlety when we use DC0 for the prediction criterion for player 2.

DC20: Let $\Gamma_{1}^{e}=\left\{d_{1}\left(\mathbf{s}_{11}\right), \neg d_{1}\left(\mathbf{s}_{12}\right)\right\}$. Then we have the following:

$$
\vdash_{E_{2} F_{2}} \mathrm{~B}_{2}\left(\hat{g}_{2}^{2}\right), \mathrm{B}_{2} \mathrm{~B}_{1}\left(\Gamma_{1}^{e}\right) \rightarrow \mathrm{B}_{2}\left(D_{2}^{20}\left(\mathbf{s}_{21}\right)\right),
$$

where $E_{2}=F_{2}=\{\epsilon,(2),(2,1)\}$. This $F_{2}$ is a unique minimal one for this sequent. From the above argument for DC $0,\{\epsilon,(2)\}$ might be expected to be a minimal one for (7.2), but actually, it is not. Let us explain the subtlety involved in (7.2) with $\Gamma_{1}^{e}$.

Suppose that player 2 assumes that 1 has the belief set $\Gamma_{1}^{s}=\left\{d_{1}\left(\mathbf{s}_{11}\right)\right\}$ rather than $\Gamma_{1}^{e}$. In this case, (7.2) breaks down, i.e.,

$$
\nvdash_{E_{2} F_{2}} \mathrm{~B}_{2}\left(\hat{g}_{2}^{2}\right), \mathrm{B}_{2} \mathrm{~B}_{1}\left(\Gamma_{1}^{s}\right) \rightarrow \mathrm{B}_{2}\left(D_{2}^{20}\left(\mathbf{s}_{21}\right)\right) .
$$

This thought sequent is unprovable whatever $F_{2}$ is. This unprovability is caused by the fact that 2 has no beliefs on strategy $\mathbf{s}_{12}$ in $\mathrm{B}_{1}\left(\Gamma_{1}^{s}\right)$, while $\mathbf{s}_{12}$ is taken into account in decision criterion $\mathscr{D}_{2}^{20}$.

From the viewpoint of player 2, we need to assume that 2 believes that 1 does not think about $\mathbf{s}_{12}$. This is formulated as $\left\{\mathrm{B}_{2} \mathrm{~B}_{1}\left(d_{1}\left(\mathbf{s}_{11}\right)\right), \mathrm{B}_{2} \neg \mathrm{B}_{1}\left(d_{1}\left(\mathbf{s}_{12}\right)\right)\right\}$ rather than $\mathrm{B}_{2} \mathrm{~B}_{1}\left(\Gamma_{1}^{e}\right)$. If this is assumed, we have

$$
\vdash_{E_{2} F_{2}} \mathrm{~B}_{2}\left(\hat{g}_{2}^{2}\right),\left\{\mathrm{B}_{2} \mathrm{~B}_{1}\left(d_{1}\left(\mathbf{s}_{11}\right)\right), \mathrm{B}_{2} \neg \mathrm{B}_{1}\left(d_{1}\left(\mathbf{s}_{12}\right)\right)\right\} \rightarrow \mathrm{B}_{2}\left(D_{2}^{20}\left(\mathbf{s}_{21}\right)\right),
$$

where $E_{2}=\{\epsilon,(2),(2,1)\}$ and $F_{2}=\{\epsilon,(1)\}$. Indeed, this $F_{2}$ is a minimal one for this thought sequent, which can be shown in the same way as in the case of DC2.

In (7.2), player 2 needs to infer, from his belief of $\mathbf{s}_{12}$ being not a default decision, that player 1 does not believe that $\mathbf{s}_{12}$ is a default decision. In this inference, we use Axiom $D$, which is expressed as the possible emptiness of $\Theta$ in the distribution rule $\left(\mathrm{B}_{i} \rightarrow \mathrm{B}_{i}\right)$. On the other hand, the result of this inference is assumed in (7.4). Therefore, $F_{2}$ for (7.4) is smaller than $F_{2}$ for (7.2).

vN (Maximin): This has a similar epistemic status to DC1 in that both require only $E_{i}=\{\epsilon,(i)\}$. However, vN differs from DC1 in being capable of recommending a decision for any game. Let $g=\left(g_{1}, g_{2}\right)$ be any game. Then it holds that for some strategy $s_{i} \in S_{i}, \vdash_{E_{i} F_{i}} \mathrm{~B}_{i}\left(\hat{g}_{i}\right) \rightarrow \mathrm{B}_{i}\left(D_{i}^{v N}\left(s_{i}\right)\right)$, where $E_{i}=F_{i}=\{\epsilon,(i)\}$. 
This $F_{i}$ is a unique minimal inferential epistemic structure for this sequent. Indeed, since $D_{i}^{v N}\left(s_{i}\right) \notin \hat{g}_{i}$, we have $(i) \in F_{i}$ by Theorem 6.3.

Here we consider the prediction-decision criterion NDC for the location game $h=\left(h_{1}, h_{2}, h_{3}\right)$, and postpone considerations of the hierarchical decision criterion HDC to the next section.

NPC: In this case, we have the following:

$$
\vdash_{E_{2} F_{2}} \mathrm{~B}_{1}\left(\hat{h}_{1}\right), \mathrm{B}_{1} \mathrm{~B}_{2}\left(\hat{h}_{2}\right), \mathrm{B}_{1} \mathrm{~B}_{3}\left(\hat{h}_{3}\right) \rightarrow \mathrm{B}_{1}\left(D_{1}^{N}\left(\mathbf{s}_{11}\right)\right),
$$

where $E_{1}=F_{1}=\{\epsilon,(1),(1,2),(1,3)\}$. This states that player 1 would choose "open his store" $\mathbf{s}_{11}$, predicting that 3 would choose $\mathbf{s}_{32}$ as an undominated strategy and 2 would choose either $\mathbf{s}_{21}$ or $\mathbf{s}_{22}$ also as an undominated one.

The inferential epistemic structure $F_{1}=\{\epsilon,(1),(1,2),(1,3)\}$ is minimal for the sequent of (7.5). Indeed, this minimality is shown in the same manner as in DC2.

\section{Transitions of the viewpoint and inner parallelisms}

In this section, we consider further reductions of decision statement (7.1), i.e., Theorem 4.1.(2). Up to now, we have kept our considerations of predictiondecision making from the viewpoint of the investigator (observer). However, before the investigator comes to his own viewpoint, he takes the viewpoint of each player $i$ as if he were player $i$. In general, the transition of the viewpoint from player $i_{m}$ to $i_{m-1}$ (or to the investigator if $m=1$ ) occurs in the epistemic distribution rule:

$$
\frac{\mathrm{B}_{\left(i_{1}, \ldots, i_{m}\right)}[\Lambda \rightarrow \Theta]}{\mathrm{B}_{\left(i_{1}, \ldots, i_{m-1}\right)}\left[\mathrm{B}_{i_{m}}(\Lambda) \rightarrow \mathrm{B}_{i_{m}}(\Theta)\right]}\left(\mathrm{B}_{i_{m}} \rightarrow \mathrm{B}_{i_{m}}\right) \text {, where }|\Theta| \leq 1 .
$$

In this section, we show that we can take the opposite route to trace this transition back. In this backward transition, we find a parallel structure to Theorem 4.1.(1), looking into the inner structures of a prediction-decision criterion. We call this parallel structure in prediction- and decision-making an inner parallelism. In Section 8.3, we will consider the implications of inner parallelisms to our basic assumption on the logical rationality of the players.

\subsection{Backward transitions and inner parallelisms}

The following are the reduction steps of the backward transitions of the viewpoint from the outward statement to a statement in a player $i$ 's mind. By an inner parallelism, we mean that after one round of the following three steps, another round of parallel three steps appear in player $i$ 's mind.

Step 0: outward $n$-person statement from the investigator's viewpoint Th.4.1.(1); 
Step 1: outward individual statement from the investigator's viewpoint Th.4.1.(2);

Step 2: individual statement from player $i$ 's viewpoint - Th.8.1.(2) \& (3);

Step 3: inner statement in player $i$ 's mind - Th.8.4.

Although the first theorem is an immediate consequence of Lemmas 6.4 and 6.5, it is worth mentioning for the understanding of our problem. In the following, we make the same setting as those for Theorem 4.1, i.e., $\Gamma_{i}$ is the set of player $i$ 's basic beliefs and $\mathscr{D}_{i}=\left\{D_{i}\left(s_{i}\right): s_{i} \in S_{i}\right\}$ is player $i$ 's prediction-decision criterion.

Theorem 8.1 (Transitions of the Viewpoint). Let $(i) \in F_{i}$. Then the following three statements are equivalent:

(1): $\vdash_{E_{i} F_{i}} \mathrm{~B}_{i}\left(\Gamma_{i}\right) \rightarrow \mathrm{B}_{i}\left(D_{i}\left(s_{i}\right)\right)$;

(2): $\vdash_{E_{i} F_{i}} \mathrm{~B}_{i}\left[\Gamma_{i} \rightarrow D_{i}\left(s_{i}\right)\right]$;

(3): $\vdash_{E_{-i} F_{-i}} \Gamma_{i} \rightarrow D_{i}\left(s_{i}\right)$.

Here $E_{-i}=\left(E_{i}\right)_{-i}=\left\{e: i \circ e \in E_{i}\right\}$ and $F_{-i}=\left(F_{i}\right)_{-i}=\left\{e: i \circ e \in F_{i}\right\}$.

The first states that the investigator derives $\mathrm{B}_{i}\left(D_{i}\left(s_{i}\right)\right)$ from $\mathrm{B}_{i}\left(\Gamma_{i}\right)$, the second that the investigator recognizes that player $i$ himself derives $D_{i}\left(s_{i}\right)$ from his basic beliefs $\Gamma_{i}$, and the third is a restatement of the second by regarding player $i$ 's viewpoint as the investigator's. Thus this theorem describes the backward transitions of the viewpoint. This backward reduction reflects the presumption that player $i$ has logically rational in the same sense as in that the investigator' logical rationality.

Theorem 8.1 can be used to obtain the equivalent unprovability statements from U1 and U2 of Section 7:

$\left(\mathrm{U} 1^{*}\right): \nvdash_{E_{2} F_{2}} \mathrm{~B}_{2}\left[\hat{g}_{2}^{2}, \mathrm{~B}_{1}\left(\hat{g}_{1}^{2}\right) \rightarrow D_{2}^{2}\left(\mathbf{s}_{22}\right)\right]$;

$\left(\mathrm{U} 2^{*}\right): \nvdash_{E_{2} F_{2}} \mathrm{~B}_{2}\left[\hat{g}_{2}^{2}, \mathrm{~B}_{1}\left(\hat{g}_{1}^{2}\right) \rightarrow \neg D_{2}^{2}\left(\mathbf{s}_{22}\right)\right]$.

Therefore, Theorem 8.1 implies that it does not matter to take either the investigator's viewpoint or player's. Note that U3 of Section 7 is changed into $\nvdash_{E_{2} F_{2}} \mathrm{~B}_{2}\left(\hat{g}_{2}^{2}\right), \mathrm{B}_{2} \mathrm{~B}_{1}\left(\hat{g}_{1}^{2}\right), \mathrm{B}_{2}\left(D_{2}^{2}\left(\mathbf{s}_{22}\right)\right) \rightarrow$ and then is reduced into $\nvdash_{E_{2} F_{2}}$ $\mathrm{B}_{2}\left[\hat{g}_{2}^{2}, \mathrm{~B}_{1}\left(\hat{g}_{1}^{2}\right), D_{2}^{2}\left(\mathbf{s}_{22}\right) \rightarrow \quad\right]$. This is equivalent to $\mathrm{U} 2 *$.

Now, we return to the general situation of Theorem 8.1, and look into the structure of $D_{i}\left(s_{i}\right)$, which is now assumed to be given as (5.1), i.e., $D_{i}\left(s_{i}\right)=$ $\bigvee_{s_{-i}} \operatorname{Pre}_{i}\left(s_{-i}\right) \wedge \bigwedge_{s_{-i}}\left(\operatorname{Pre}_{i}\left(s_{-i}\right) \supset \operatorname{Res}_{i}\left(s_{i} \mid s_{-i}\right)\right)$. Theorem 8.2 is also immediate, but is stated explicitly. Recall $\operatorname{Pre}_{i}\left(s_{-i}\right)=\bigwedge_{j \neq i} \operatorname{Pre}_{i j}\left(s_{j}\right)$.

Theorem 8.2. Let $(i) \in F_{i}$. Then $\vdash_{E_{i} F_{i}} \mathrm{~B}_{i}\left(\Gamma_{i}\right) \rightarrow \mathrm{B}_{i}\left(D_{i}\left(s_{i}\right)\right)$ if and only if the following two hold:

(1): $\vdash_{E_{-i} F_{-i}} \Gamma_{i} \rightarrow \bigvee_{s_{-i}} \operatorname{Pre}_{i}\left(s_{-i}\right)$;

(2): $\vdash_{E_{-i} F_{-i}} \Gamma_{i} \rightarrow \bigwedge_{s_{-i}}\left(\operatorname{Pre}_{i}\left(s_{-i}\right) \supset \operatorname{Res}_{i}\left(s_{i} \mid s_{-i}\right)\right)$. 
Proof. Suppose (1) and (2). It follows from (1) and (2) that $\vdash_{E_{-i} F_{-i}} \Gamma_{i} \rightarrow$ $\bigvee_{s_{-i}} \operatorname{Pre}_{i}\left(s_{-i}\right) \wedge \bigwedge_{s_{-i}}\left(\operatorname{Pre}_{i}\left(s_{-i}\right) \supset \operatorname{Res}_{i}\left(s_{i} \mid s_{-i}\right)\right)$, i.e., $\vdash_{E_{-i} F_{-i}} \Gamma_{i} \rightarrow D_{i}\left(s_{i}\right)$. This is equivalent to $\vdash_{E_{i} F_{i}} \mathrm{~B}_{i}\left[\Gamma_{i} \rightarrow D_{i}\left(s_{i}\right)\right]$ by Lemma 6.5. Then, by $\left(\mathrm{B}_{i} \rightarrow \mathrm{B}_{i}\right)$, we have $\vdash_{E_{i} F_{i}} \mathrm{~B}_{i}\left(\Gamma_{i}\right) \rightarrow \mathrm{B}_{i}\left(D_{i}\left(s_{i}\right)\right)$.

Suppose $\vdash_{E_{i} F_{i}} \mathrm{~B}_{i}\left(\Gamma_{i}\right) \rightarrow \mathrm{B}_{i}\left(D_{i}\left(s_{i}\right)\right)$. This is equivalent to $\vdash_{E_{-i} F_{-i}} \Gamma_{i} \rightarrow$ $D_{i}\left(s_{i}\right)$ by Theorem 8.1, i.e., $\vdash_{E_{-i} F_{-i}} \Gamma_{i} \rightarrow\left(\bigvee_{s_{-i}} \operatorname{Pre}_{i}\left(s_{-i}\right)\right) \wedge \bigwedge_{s_{-i}}\left(\operatorname{Pre}_{i}\left(s_{-i}\right) \supset\right.$ $\operatorname{Res}_{i}\left(s_{i} \mid s_{-i}\right)$ ). This implies (1) and (2).

This theorem states that the investigator derives $\mathrm{B}_{i}\left(D_{i}\left(s_{i}\right)\right)$ from $\mathrm{B}_{i}\left(\Gamma_{i}\right)$ if and only if player $i$ derives, in his mind, $\bigvee_{s_{-i}} \operatorname{Pre}_{i}\left(s_{-i}\right)--$ the existence of predicted strategies for the other players - - and $\bigwedge_{s_{-i}}\left(\operatorname{Pre}_{i}\left(s_{-i}\right) \supset\right.$ $\left.\operatorname{Res}_{i}\left(s_{i} \mid s_{-i}\right)\right)--$ the appropriateness of his decision $s_{i}$ to the predicted strategies. In fact, the existence of his predictions has a similar status to (4.7), i.e., $\Gamma_{0}, \mathrm{~B}_{1}\left(\Gamma_{1}\right), \ldots, \mathrm{B}_{n}\left(\Gamma_{n}\right) \rightarrow \bigvee_{s_{1}} \mathrm{~B}_{1}\left(D_{1}\left(s_{1}\right)\right) \wedge \ldots \wedge \bigvee_{s_{n}} \mathrm{~B}_{n}\left(D_{n}\left(s_{n}\right)\right)$. We can regard (4.7) as the predictions made by the investigator. In the mind of player $i$, player $i$ makes a similar prediction. We call this parallel structure an inner parallelism.

To explicate this inner parallelism more, we look into Theorem 8.2.(2). First, we have the following lemma.

Lemma 8.3. If (a) $\vdash_{E_{-i} F_{-i}} \Gamma_{i} \rightarrow \bigvee_{s_{-i}} \bigwedge_{j \neq i} \operatorname{Pre}_{i j}\left(s_{j}\right)$, then (b) $\vdash_{E_{-i} F_{-i}} \Gamma_{i} \rightarrow$ $\bigwedge_{j \neq i} \bigvee_{s_{j}} \operatorname{Pre}_{i j}\left(s_{j}\right)$.

Proof. Suppose (a). Let $t_{-i}$ be an arbitrary element in $S_{-i}$. Using successively $(\bigwedge \rightarrow)$ and $(\rightarrow \bigvee)$, we have $\vdash_{E_{-i} F_{-i}} \bigwedge_{j \neq i} \operatorname{Pre}_{i j}\left(t_{j}\right) \rightarrow \bigvee_{s_{k}} \operatorname{Pre}_{i k}\left(s_{k}\right)$ for all $k \neq i$. Then $\vdash_{E_{-i} F_{-i}} \bigwedge_{j \neq i} \operatorname{Pre}_{i j}\left(t_{j}\right) \rightarrow \bigwedge_{j \neq i} \bigvee_{s_{j}} \operatorname{Pre}_{i j}\left(s_{j}\right)$ by $(\rightarrow \bigwedge)$. Since $t_{-i}$ is arbitrary in $S_{-i}$, we have, by $(\bigvee \rightarrow), \vdash_{E_{-i} F_{-i}} \bigvee_{s_{-i}} \bigwedge_{j \neq i} \operatorname{Pre}_{i j}\left(s_{j}\right) \rightarrow \bigwedge_{j \neq i} \bigvee_{s_{j}} \operatorname{Pre}_{i j}\left(s_{j}\right)$. Using this conclusion and the supposition of the lemma, we have

$$
\frac{\Gamma_{i} \rightarrow \bigvee_{s_{-i}} \bigwedge_{j \neq i} \operatorname{Pre}_{i j}\left(s_{j}\right) \quad \bigvee_{s_{-i}} \bigwedge_{j \neq i} \operatorname{Pre}_{i j}\left(s_{j}\right) \rightarrow \bigwedge_{j \neq i} \bigvee_{s_{j}} \operatorname{Pre}_{i j}\left(s_{j}\right)}{\Gamma_{i} \rightarrow \bigwedge_{j \neq i} \bigvee_{s_{j}} \operatorname{Pre}_{i j}\left(s_{j}\right)} \text { (Cut) }
$$

Unless we assume further structures on the belief set $\Gamma_{i}$ and prediction criterion $\operatorname{Pre}_{i j}\left(s_{j}\right)$, we could not go further than (b) of Lemma 8.3. However, we find certain natural assumptions on $\Gamma_{i}$ and $\operatorname{Pre}_{i j}\left(s_{j}\right)$ from the viewpoint of player $i$. First, we make the following assumption on the belief set $\Gamma_{i}$ of player $i$ :

$$
\Gamma_{i} \text { is written as } \Gamma_{i 0} \cup \bigcup_{j \neq i} \mathrm{~B}_{j}\left(\Gamma_{i j}\right),
$$

where $\Gamma_{i 0}$ consists of nonepistemic formulae and $\Gamma_{i j}$ is any finite set of formulae for $j \neq i$. Second, we make the following assumption on the prediction criterion $\mathscr{P}_{i}=\left\{\operatorname{Pre}_{i j}\left(s_{j}\right): s_{j} \in S_{j}\right.$ and $\left.j \in N-\{i\}\right\}:$

$$
\text { each } \operatorname{Pre}_{i j}\left(s_{j}\right) \text { is expressed as } \mathrm{B}_{j}\left(\operatorname{Pre}_{i j}^{0}\left(s_{j}\right)\right) \text {. }
$$

Here, $\operatorname{Pre}_{i j}^{0}\left(s_{j}\right)$ may be any formula for $j \neq i$. For example, the prediction-decision criterion DC2 takes this form. For simplicity, we assume (8.2) for all players. 
For this reason, (8.2) is not fully satisfied in the consideration of criterion HDC below, but the modification is straightforward.

Under the assumptions (8.1) and (8.2), (b) of Lemma 8.3 is expressed as

$$
\vdash_{E_{-i} F_{-i}} \Gamma_{i 0} \cup \bigcup_{j \neq i} \mathrm{~B}_{j}\left(\Gamma_{i j}\right) \rightarrow \bigwedge_{j \neq i} \bigvee_{s_{j}} \mathrm{~B}_{j}\left(\operatorname{Pre}_{i j}^{0}\left(s_{j}\right)\right) .
$$

Now, we have an apparent parallelism between (4.7) and (8.3). That is, (8.3) is an outward description of prediction makings and is stated as if player $i$ were the investigator. An only difference from (4.7) is that player $i$ himself is excluded in (8.3), since he is predicting what the others would choose. If we have equivalence between the two statements of Lemma 8.3, then our parallelism is complete. The following theorem states this equivalence under (8.1) and (8.2).

Theorem 8.4 (Inner parallelism). Assume (8.1) and (8.2), and that $\Gamma_{i}$ is consistent in $\mathrm{GL}_{E_{-i} F_{-i}}$. Then (b) of Lemma 8.3 is equivalent to (a), which is further equivalent to that for all $j \neq i$,

$$
\vdash_{E_{-i} F_{-i}} \mathrm{~B}_{j}\left(\Gamma_{i j}\right) \rightarrow \mathrm{B}_{j}\left(\operatorname{Pre}_{i j}^{0}\left(s_{j}\right)\right) \text { for some } s_{j} \in S_{j} .
$$

Proof. Suppose (8.4) for all $j \neq i$. Then $\vdash_{E_{-i} F_{-i}} \Gamma_{i} \rightarrow \bigwedge_{j \neq i} \mathrm{~B}_{j}\left(\operatorname{Pre}_{i j}^{0}\left(s_{j}\right)\right)$ for some $s_{-i} \in S_{-i}$. Hence $\vdash_{E_{-i} F_{-i}} \Gamma_{i} \rightarrow \bigvee_{s_{-i}} \bigwedge_{j \neq i} \mathrm{~B}_{j}\left(\operatorname{Pre}_{i j}^{0}\left(s_{j}\right)\right)$, which is (a) of Lemma 8.3. It remains to show that the (b) of Lemma 8.3 implies (8.4).

Suppose $\vdash_{E_{-i} F_{-i}} \Gamma_{i} \rightarrow \bigwedge_{j \neq i} \bigvee_{s_{j}} \operatorname{Pre}_{i j}\left(s_{j}\right)$. Then $\vdash_{E_{-i} F_{-i}} \Gamma_{i} \rightarrow \bigvee_{s_{j}} \mathrm{~B}_{j}\left(\operatorname{Pre}_{i j}^{0}\left(s_{j}\right)\right)$ for all $j \neq i$. Let $k \neq i$ be an arbitrary player. Since $\vdash_{E_{-i} F_{-i}} \Gamma_{i 0} \cup \bigcup_{j \neq i} \mathrm{~B}_{j}\left(\Gamma_{i j}\right) \rightarrow$ $\bigvee_{s_{k}} \mathrm{~B}_{k}\left(\operatorname{Pre}_{i k}^{0}\left(s_{k}\right)\right)$ by (8.1) and (8.2), By Theorem 6.1, we have $\vdash_{E_{-i} F_{-i}} \mathrm{~B}_{k}\left(\Gamma_{i k}\right) \rightarrow$ $\bigvee_{s_{k}} \mathrm{~B}_{k}\left(\operatorname{Pre}_{i k}^{0}\left(s_{k}\right)\right)$. By Theorem 6.2, we have $\vdash_{E_{-i} F_{-i}} \mathrm{~B}_{k}\left(\Gamma_{i k}\right) \rightarrow \mathrm{B}_{k}\left(\operatorname{Pre}_{i k}^{0}\left(s_{k}\right)\right)$ for some $s_{k} \in S_{k}$. Thus, we have (8.4).

Notice the parallelism between (8.4) and

$$
\vdash_{E F} \mathrm{~B}_{i}\left(\Gamma_{i}\right) \rightarrow \mathrm{B}_{i}\left(D_{i}\left(s_{i}\right)\right) \text { for some } s_{i} \in S_{i}
$$

Statement (8.4) states that the investigator thinks that player $j$ can derive $\operatorname{Pre}_{i j}^{0}\left(s_{j}\right)$ from $\mathrm{B}_{j}\left(\Gamma_{i j}\right)$. $\operatorname{Pre}_{i j}^{0}\left(s_{j}\right)$ means the decision for player $j$ predicted by player $i$. Therefore, this is essentially the same as $D_{i}\left(s_{i}\right)$ from the investigator's viewpoint. We can repeat another round of Steps 1-3 from (8.4).

\subsection{Further reductions}

The reduction of decision statements eventually leads us to look into the inner structure of each prediction-decision criterion. We have started the discourse of reductions from Theorem 8.1.(1), and now, we can repeat the same discourse from (8.4). Indeed, applying the argument for Theorem 8.1 to (8.4), we obtain

$$
\vdash_{E_{-i} F_{-i}} \mathrm{~B}_{j}\left[\Gamma_{i j} \rightarrow \operatorname{Pre}_{i j}^{0}\left(s_{j}\right)\right]
$$


if $(j) \in F_{-i}$.

The parallelism can be even more explicit if we specify the prediction criterion $\mathrm{B}_{j}\left(\operatorname{Pre}_{i j}^{0}\left(s_{j}\right)\right)$ more. Now, we assume that each $\operatorname{Pre}_{i j}^{0}\left(s_{j}\right)$ is written as the same form of (5.1), that is, it is defined by another prediction criterion $\mathscr{P}_{j k}^{i}=\left\{\operatorname{Pre}_{j k}^{i}\left(s_{k}\right): s_{k} \in S_{k}\right\}$ and valuation relation $\mathscr{R}_{j}^{i}=\left\{\operatorname{Res}_{j}^{i}\left(s_{j} \mid s_{-j}\right): s \in S\right\}:$

$$
\operatorname{Pre}_{i j}^{0}\left(s_{j}\right)=\bigvee_{s_{-j}} \operatorname{Pre}_{j}^{i}\left(s_{-j}\right) \wedge \bigwedge_{s_{-j}}\left(\operatorname{Pre}_{j}^{i}\left(s_{-j}\right) \supset \operatorname{Res}_{j}^{i}\left(s_{j} \mid s_{-j}\right)\right),
$$

where $\operatorname{Pre}_{j}^{i}\left(s_{-j}\right)=\bigwedge_{k \neq j} \operatorname{Pre}_{j k}^{i}\left(s_{k}\right)$. We note that $\operatorname{Pre}_{j k}^{i}\left(s_{k}\right)$ and $\operatorname{Res}_{j}^{i}\left(s_{j} \mid s_{-j}\right)$ are "subjective" ones in the mind of player $i$, and that they may differ from the "true" $\operatorname{Pre}_{j k}\left(s_{k}\right)$ and $\operatorname{Res}_{j}\left(s_{j} \mid s_{-j}\right)$.

Now, if $(j) \in F_{-i}$, then (8.4) becomes

$$
\vdash_{E_{-i j} F_{-i j}} \Gamma_{i j} \rightarrow \bigvee_{s_{-j}} \operatorname{Pre}_{j}^{i}\left(s_{-j}\right) \wedge \bigwedge_{s_{-j}}\left(\operatorname{Pre}_{j}^{i}\left(s_{-j}\right) \supset \operatorname{Res}_{j}^{i}\left(s_{j} \mid s_{-j}\right)\right),
$$

where $E_{-i j}=\left(E_{-i}\right)_{-j}$ and $F_{-i j}=\left(F_{-i}\right)_{-j}$. Hence, we can repeat Theorem 8.2, Lemma 8.3 and Theorem 8.4 for (8.7). This repeating process is summarized in the steps in the beginning of this section.

The inner parallelism is trivial in our examples of prediction-decision criteria, except for criterion HDC in the location game LG of Section 2. Here, we look at HDC briefly.

HDC: First, it holds that

$$
\vdash_{E_{1} F_{1}} \mathrm{~B}_{1}\left(\Gamma_{1}\right) \rightarrow \mathrm{B}_{1}\left(D_{1}^{H}\left(\mathbf{s}_{11}\right)\right),
$$

where $\Gamma_{1}=\hat{h}_{1} \cup \mathrm{B}_{2}\left(\hat{h}_{2}\right) \cup \mathrm{B}_{2} \mathrm{~B}_{3}\left(\hat{h}_{3}\right)$ and $E_{1}=F_{1}=\{\epsilon,(1),(1,2),(1,2,3)\}$. This $F_{1}$ is a unique minimal inferential epistemic structure. However, our present concern is the deduction of (8.8) into the following:

(a1): $\vdash_{E_{1} F_{1}} \mathrm{~B}_{1}\left[\Gamma_{1} \rightarrow D_{1}^{H}\left(\mathbf{s}_{11}\right)\right]$;

(a2): $\vdash_{E_{-1} F_{-1}} \Gamma_{1} \rightarrow D_{1}^{H}\left(\mathbf{s}_{11}\right)$.

Thus, the player's inner viewpoint is regarded as the investigator's. Recall that $D_{1}^{H}\left(s_{1}\right)$ is given as $\bigvee_{s_{-1}} \operatorname{Pre}_{1}^{H}\left(s_{-1}\right) \wedge \bigwedge_{s_{-1}}\left(\operatorname{Pre}_{1}^{H}\left(s_{-1}\right) \supset \operatorname{Best}_{1}\left(s_{1} \mid s_{-1}\right)\right)$. Then (a2) is reduced into

(b1): $\vdash_{E_{-1} F_{-1}} \Gamma_{1} \rightarrow \bigvee_{s_{-1}} \operatorname{Pre}_{1}^{H}\left(s_{-1}\right)$;

(b2): $\vdash_{E_{-1} F_{-1}} \Gamma_{1} \rightarrow \bigwedge_{s_{-1}}\left(\operatorname{Pre}_{1}^{H}\left(s_{-1}\right) \supset \operatorname{Best}_{1}\left(\mathbf{s}_{11} \mid s_{-1}\right)\right)-$ Theorem 8.2.

Then (b1) is further reduced into

(c) $: \vdash_{E_{-1} F_{-1}} \mathrm{~B}_{2}\left(\hat{h}_{2}\right), \mathrm{B}_{2} \mathrm{~B}_{3}\left(\hat{h}_{3}\right) \rightarrow \operatorname{Pre}_{12}^{H}\left(s_{2}\right)$ for some $s_{2} \in S_{2}-$ Theorem 8.4.

Since $\operatorname{Pre}_{12}^{H}\left(s_{2}\right)=B_{2}\left(\bigvee_{s_{-2}}\left(P_{21}^{0}\left(s_{1}\right) \wedge \mathrm{B}_{3}\left(\operatorname{Dom}_{3}\left(s_{3}\right)\right)\right) \wedge \bigwedge_{s_{-2}}\left(P_{21}^{0}\left(s_{1}\right) \wedge \mathrm{B}_{3}\left(\operatorname{Dom}_{3}\left(s_{3}\right)\right)\right.\right.$

$\left.\supset \operatorname{Best}_{2}\left(s_{2} \mid s_{-2}\right)\right)$, we can repeat a parallel reduction from statement (c). 


\subsection{Basic assumption on $G L_{E F}$ for the inner parallelisms}

Here, let us consider basic assumptions for the inner parallelisms. There are two basic assumptions relevant for them. First, a prediction-decision criterion in question has an inner parallelism in the sense that prediction about another player's behavior takes the form of a prediction-decision criterion. This inner parallelism must be apparent in the previous two subsections. The other is a basic assumption for epistemic logic $\mathrm{GL}_{E F}$, which enables us to discuss the inner parallelisms.

Let $\left(i_{1}, \ldots, i_{k}, \ldots, i_{m}\right)$ be any epistemic status in $F$. Recall the basic assumption that player $i_{1}$ has the logical rationality same as the investigator's, which are reflected in Lemmas 6.4 and 6.5. In fact, this basic assumption is made for any (imagined) player $i_{k}$ in $\left(i_{1}, \ldots, i_{k}, \ldots, i_{m}\right)$. That is, player $i_{k}$ in the mind of $i_{k-1} \ldots$ in the mind of $i_{1}$ has the same logical rationality as the investigator. Technically speaking, player $i_{k}$ 's logical rationality is represented by the logic $\mathrm{GL}_{E^{k} F^{k}}$, where $E^{k}=\left\{e:\left(i_{1}, \ldots, i_{k}\right) \circ e \in E\right\}$ and $F^{k}=\left\{e:\left(i_{1}, \ldots, i_{k}\right) \circ e \in F\right\}$. If $\mathrm{GL}_{E^{k} F^{k}}$ is regarded as part of $\mathrm{GL}_{E F}$, then it describes the logical rationality of $i_{k}$ in the mind of $i_{k-1} \ldots$ in the mind of $i_{1}$. On the other hand, if $\mathrm{GL}_{E^{k} F^{k}}$ is considered alone, it describes the investigator's logical reasoning. Thus, we have treated the players even appearing in the minds of players as well as the investigator in the same manner. This treatment guarantees the inner parallelisms.

\section{Further developments}

In this section, we give two remarks on further developments of our logico-game analysis. Specifically, we consider a compound prediction-decision criterion and a compound one with a last-resort default decision. In Section 9.1, we take the prediction-decision criteria DC1 and DC2 of Section 2.2 as composing subcriteria, though we can discuss compound criteria in a more general manner. More extensive treatments will be given in a future paper.

\subsection{Compound decision criteria}

Suppose that player $i$ has decision criteria $\mathscr{D}_{i}{ }^{1}$ and $\mathscr{D}_{i}^{2}$. We formulate the new compound criterion $\mathscr{D}_{i}^{c}=\left\{D_{i}^{c}\left(s_{i}\right): s_{i} \in S_{i}\right\}$ as follows:

$$
D_{i}^{c}\left(s_{i}\right)=D_{i}^{1}\left(s_{i}\right) \vee D_{i}^{2}\left(s_{i}\right) \text { for all } s_{i} \in S_{i} .
$$

This recommends a strategy if at least one of $\mathscr{D}_{i}^{1}$ and $\mathscr{D}_{i}^{2}$ does it.

Theorem 4.1 holds for the above compound criterion $\mathscr{D}_{i}^{c}$. Hence, we can focus on the individual decision making of player $i$. Here we look the case of $i=2$. Let $g=\left(g_{1}, g_{2}\right)$ be a two-person game, and let the belief set of player $i$ be given as $\Gamma_{i}(\hat{g})=\hat{g}_{i} \cup \mathrm{B}_{j}\left(\hat{g}_{j}\right)$, where $i, j=1,2(i \neq j)$. We would like to find a minimal $F_{2}(g)$ so that 


$$
\vdash_{E_{2} F_{2}(g)} \mathrm{B}_{2}\left(\Gamma_{2}(\hat{g})\right) \rightarrow \bigvee_{s_{2}} \mathrm{~B}_{2}\left(D_{2}^{c}\left(s_{2}\right)\right),
$$

where $E_{2}=\{\epsilon,(2),(2,1)\}$. A minimal inferential epistemic structure $F_{2}(\hat{g})$ for (9.2) depends upon game $g$. When we look at game $g^{1}$ or $g^{2}$, we have the following result. We omit its proof.

Theorem 9.1 (Minimal epistemic structures in games $g^{1}$ and $g^{2}$ ).

(1): Let $g=g^{1}$. The minimal $F_{2}\left(\hat{g}^{1}\right)$ for (9.2) is $\{\epsilon,(2)\}$.

(2): Let $g=g^{2}$. The minimal $F_{2}\left(\hat{g}^{2}\right)$ for $(9.2)$ is $\{\epsilon,(2),(2,1)\}$.

In all the previous examples, a minimal inferential epistemic structure for a sequent is uniquely determined. Here we give one counterexample by considering the compound decision criterion $\mathscr{D}_{1}^{H N}=\left\{D_{1}^{H N}\left(s_{1}\right): s_{1} \in S_{1}\right\}$ of $\mathscr{D}_{1}^{H}$ and $\mathscr{D}_{1}^{N}$ for the location game LG, where $D_{1}^{H N}\left(s_{1}\right)=D_{1}^{H}\left(s_{1}\right) \vee D_{1}^{N}\left(s_{1}\right)$ for $s_{1} \in S_{1}$. Then it follows from (7.5) and (8.8) that

$$
\vdash_{E F} \mathrm{~B}_{1}\left(\hat{h}_{1}\right), \mathrm{B}_{1} \mathrm{~B}_{2}\left(\hat{h}_{2}\right), \mathrm{B}_{1} \mathrm{~B}_{3}\left(\hat{h}_{3}\right), \mathrm{B}_{1} \mathrm{~B}_{2} \mathrm{~B}_{3}\left(\hat{h}_{3}\right) \rightarrow \bigvee_{s_{1}} D_{1}^{H N}\left(s_{1}\right),
$$

where $E=\{\epsilon,(1),(1,2),(1,3),(1,2,3)\}$ and $F$ is an epistemic structure including $\{\epsilon,(1),(1,2),(1,3)\}$ or $\{\epsilon,(1),(1,2),(1,2,3)\}$. We can prove the following theorem, whose proof is omitted.

Theorem 9.2 (Minimal epistemic structures in location game). There are exactly two minimal epistemic structures $F$ for $(9.3)$, which are $\{\epsilon,(1),(1,2),(1,3)\}$ and $\{\epsilon,(1),(1,2),(1,2,3)\}$.

\subsection{The last-resort default decision}

Recall that the game $g^{4}=\left(g_{1}^{4}, g_{2}^{4}\right)$ of Table 4 has no dominant strategies and no Nash equilibria. Then (9.2) does not hold for this game as far as $\Gamma_{2}\left(\hat{g}^{4}\right)=$ $\hat{g}_{2}^{4} \cup \mathrm{B}_{1}\left(\hat{g}_{1}^{4}\right)$ is adopted. The pure default decision criterion can be applied to this game. However, the following method may be more typical than applying the default decision directly to this game: First, one considers non-default decision criteria, and if those criteria give no decisions, then a default is applied. This idea can be formulated as adding the last-resort default decision to the compound criterion.

The compound decision criterion $\mathscr{D}_{i}^{C}=\left\{D_{i}^{C}\left(s_{i}\right): s_{i} \in S_{i}\right\}$ of composing criteria $\mathscr{D}_{i k}=\left\{D_{i k}\left(s_{i}\right): s_{i} \in S_{i}\right\}, k=1, \ldots, m$, is given simply as

$$
D_{i}^{C}\left(s_{i}\right)=D_{i 1}\left(s_{i}\right) \vee \ldots \vee D_{i m}\left(s_{i}\right) \text { for all } s_{i} \in S_{i} .
$$

Composing criteria of $\mathscr{D}_{i 1}, \ldots, \mathscr{D}_{i m}$ themselves may be compound criteria of some other composing criteria.

The compound decision criterion $\mathscr{D}^{C l}=\left\{D_{i}^{C l}\left(s_{i}\right): s_{i} \in S_{i}\right\}$ with the lastresort default is formulated as 


$$
D_{i}^{C l}\left(s_{i}\right)=D_{i}^{C}\left(s_{i}\right) \vee d_{i}\left(s_{i}\right) \text { for all } s_{i} \in S_{i} .
$$

This criterion itself is formulated as a compound criterion.

As we needed to assume that the belief set of player $i$ includes some predetermined default decision for (4.16), we need some assumption on the belief set of player $i$ to make (9.5) workable. The idea of the last-resort default decision is: if player $i$ verifies that none of his strategies satisfy any of his non-default decision criteria, then he would use the predetermined default decision $\mathbf{s}_{i 1}$. This idea is expressed as

$$
\mathrm{B}_{i}\left(\neg \bigvee_{s_{i}} D_{i}^{C}\left(s_{i}\right) \supset d_{i}\left(\mathbf{s}_{i 1}\right)\right)
$$

The antecedent of the inside of $\mathrm{B}_{i}$ of (9.6) means the negation of criterion $\mathscr{D}_{i}^{C}$ for any strategy. Thus, $\neg \bigvee_{s_{i}} D_{i}^{C}\left(s_{i}\right)$ is a key for (9.6).

We assume that the belief set $\Gamma_{i}$ of player $i$ is given as $\Gamma_{i}(\hat{g}) \cup\left\{\neg \bigvee_{s_{i}} D_{i}^{C}\left(s_{i}\right) \supset\right.$ $\left.\left.\left.d_{i}\left(\mathbf{s}_{i 1}\right)\right)\right\}=\hat{g}_{i} \cup \bigcup_{j \neq i} \mathrm{~B}_{j}\left(\Gamma_{i j}\right) \cup\left\{\neg \bigvee_{s_{i}} D_{i}^{C}\left(s_{i}\right) \supset d_{i}\left(\mathbf{s}_{i 1}\right)\right)\right\}$, where $\Gamma_{i j}$ is any finite set of formulae. Then the derivation of the last-resort default decision is equivalent to the derivation of the negation of the other criteria. Namely,

Theorem 9.3. Let $(i) \in F_{i}$. Suppose that $\mathrm{B}_{i}\left(\Gamma_{i}\right)=\mathrm{B}_{i}\left(\Gamma_{i}(\hat{g})\right) \cup\left\{\mathrm{B}_{i}\left(\neg \bigvee_{s_{i}} D_{i}^{C}\left(s_{i}\right)\right.\right.$ $\left.\left.\supset d_{i}\left(\mathbf{s}_{i 1}\right)\right)\right\}$ is consistent in $\mathrm{GL}_{E_{i} F_{i}}$. Then the following two statements are equivalent:

(1): $\vdash E_{i} F_{i} \mathrm{~B}_{i}\left(\Gamma_{i}\right) \rightarrow \mathrm{B}_{i}\left(d_{i}\left(\mathbf{s}_{i 1}\right)\right)$;

(2): $\vdash_{E_{i} F_{i}} \mathrm{~B}_{i}\left(\Gamma_{i}\right) \rightarrow \mathrm{B}_{i}\left(\neg \bigvee_{s_{i}} D_{i}^{C}\left(s_{i}\right)\right)$.

Thus, player $i$ uses the default decision $\mathbf{s}_{i 1}$ if and only if he proves that none of the other criteria recommends a decision. This equivalence reflects the use of a default decision in our ordinary life.

The derivation of (1) from (2) needs some steps, but the converse is essential and is proved below. Either (1) or (2) is stated from the viewpoint of the investigator, but is equivalent to the following statement from the viewpoint of player $i$ :

(3): $\vdash_{E_{-i} F_{-i}} \Gamma_{i} \rightarrow \neg \bigvee_{s_{i}} D_{i}^{C}\left(s_{i}\right)$.

The equivalence between (2) and (3) are guaranteed by Lemmas 6.4 and 6.5.

In the proof of Theorem 9.3 and in the Appendix, we use the following terminology: a side formula of an operational inference rule $I$ is one to be changed in the upper sequent, and a principal formula of $I$ is one changed in the lower sequent. For example, in

$$
\frac{\Gamma \rightarrow \Theta, A \quad B, \Gamma \rightarrow \Theta}{A \supset B, \Gamma \rightarrow \Theta}(\supset \rightarrow)
$$

$A$ and $B$ are side formulae and $A \supset B$ is the principal formula. When $I$ is the distribution rule

$$
\frac{\mathrm{B}_{e \circ i}[\Gamma \rightarrow \Theta]}{\mathrm{B}_{e}\left[\mathrm{~B}_{i}(\Gamma) \rightarrow \mathrm{B}_{i}(\Theta)\right]}\left(\mathrm{B}_{i} \rightarrow \mathrm{B}_{i}\right),
$$


all formulae in the upper sequent are side formulae and all in the lower sequent are principal formulae. When $I$ is a thinning (Th), a formula added in the lower sequent is called a thinning formula.

Proof of Theorem 9.3. We prove that (1) implies (2). Suppose (1). That is,

$$
\vdash_{E_{i} F_{i}} \mathrm{~B}_{i}\left(\hat{g}_{i}\right), \bigcup_{j \neq i} \mathrm{~B}_{i} \mathrm{~B}_{j}\left(\Gamma_{i j}\right), \mathrm{B}_{i}\left(\neg \bigvee_{s_{i}} D_{i}^{C}\left(s_{i}\right) \supset d_{i}\left(\mathbf{s}_{i 1}\right)\right) \rightarrow \mathrm{B}_{i}\left(d_{i}\left(\mathbf{s}_{i 1}\right)\right) .
$$

By $(i) \in F_{i}$, we can apply Lemmas 6.4 and 6.5 to this sequent, and have

$$
\vdash_{E_{-i} F_{-i}} \hat{g}_{i}, \bigcup_{j \neq i} \mathrm{~B}_{j}\left(\Gamma_{i j}\right), \neg \bigvee_{s_{i}} D_{i}^{C}\left(s_{i}\right) \supset d_{i}\left(\mathbf{s}_{i 1}\right) \rightarrow d_{i}\left(\mathbf{s}_{i 1}\right) .
$$

By the definition of $\hat{g}_{i}, \hat{g}_{i}$ is expressed as $\hat{g}_{i}=\hat{g}_{i}^{+} \cup\left\{\neg A: A \in \hat{g}_{i}^{-}\right\}$with $\hat{g}_{i}^{+} \cap \hat{g}_{i}^{-}=\emptyset$. Also, each formula in $\hat{g}_{i}^{+}$and $\hat{g}_{i}^{-}$is atomic. Then we have

$$
\vdash_{E_{-i} F_{-i}} \hat{g}_{i}^{+}, \bigcup_{j \neq i} \mathrm{~B}_{j}\left(\Gamma_{i j}\right), \neg \bigvee_{s_{i}} D_{i}^{C}\left(s_{i}\right) \supset d_{i}\left(\mathbf{s}_{i 1}\right) \rightarrow d_{i}\left(\mathbf{s}_{i 1}\right), \hat{g}_{i}^{-} .
$$

Let $\Delta=\hat{g}_{i}^{+} \cup \bigcup_{j \neq i} \mathrm{~B}_{j}\left(\Gamma_{i j}\right)$ and $\Lambda=\left\{d_{i}\left(\mathbf{s}_{i 1}\right)\right\} \cup \hat{g}_{i}^{-}$. By the Cut-Elimination Theorem (Theorem 4.3), we have a cut-free proof $P$ of $\Delta, \neg \bigvee_{s_{i}} D_{i}^{C}\left(s_{i}\right) \supset d_{i}\left(\mathbf{s}_{i 1}\right) \rightarrow \Lambda$. Note that $\hat{g}_{i}^{+}$and $\hat{g}_{i}^{-}$consist of atomic formulae with $\hat{g}_{i}^{+} \cap \hat{g}_{i}^{-}=\emptyset$.

First, we show that $\Delta \rightarrow \Lambda$ is not provable. Then, by Theorem 6.1, we have $\vdash_{E_{-i} F_{-i}} \hat{g}_{i}^{+} \rightarrow d_{i}\left(\mathbf{s}_{i 1}\right), \hat{g}_{i}^{-}$or $\vdash_{E_{-i} F_{-i}} \bigcup_{j \neq i} \mathrm{~B}_{j}\left(\Gamma_{i j}\right) \rightarrow$. The former is equivalent to $\vdash_{E_{-i} F_{-i}} \hat{g}_{i} \rightarrow d_{i}\left(\mathbf{s}_{i 1}\right)$, which is not the case. The latter is also not the case by the consistency assumption of the theorem. Therefore, $\Delta^{\prime} \rightarrow \Lambda^{\prime}$ is not provable for any $\Delta^{\prime} \subseteq \Delta$ and $\Lambda^{\prime} \subseteq \Lambda$. For otherwise, $\Delta \rightarrow \Lambda$ would be provable by (Th).

Looking at the formulae in $\Delta, \neg \bigvee_{s_{i}} D_{i}^{C}\left(s_{i}\right) \supset d_{i}\left(\mathbf{s}_{i 1}\right) \rightarrow \Lambda$, we find that the lowermost inference in the proof $P$ is either (Th) or $(\supset \rightarrow)$. If it is (Th), the upper sequent is $\Delta^{\prime}, \neg \bigvee_{s_{i}} D_{i}^{C}\left(s_{i}\right) \supset d_{i}\left(\mathbf{s}_{i 1}\right) \rightarrow \Lambda^{\prime}$ for some $\Delta^{\prime} \subseteq \Delta$ and $\Lambda^{\prime} \subseteq \Lambda$, which follows from the conclusion of the above paragraph. We can repeat this argument until we meet $(\supset \rightarrow)$. Hence we can assume that for some $\Delta^{\prime} \subseteq \Delta$ and $\Lambda^{\prime} \subseteq \Lambda, \Delta^{\prime}, \neg \bigvee_{s_{i}} D_{i}^{C}\left(s_{i}\right) \supset d_{i}\left(\mathbf{s}_{i 1}\right) \rightarrow \Lambda^{\prime}$ is the lower sequent of $(\supset \rightarrow)$. Then $(\supset \rightarrow)$ is expressed as

$$
\frac{\Delta^{\prime}, \rightarrow \Lambda^{\prime}, \neg \bigvee_{s_{i}} D_{i}^{C}\left(s_{i}\right) \quad \Delta^{\prime}, d_{i}\left(\mathbf{s}_{i 1}\right) \rightarrow \Lambda^{\prime}}{\Delta^{\prime}, \neg \bigvee_{s_{i}} D_{i}^{C}\left(s_{i}\right) \supset d_{i}\left(\mathbf{s}_{i 1}\right) \rightarrow \Lambda^{\prime}}(\supset \rightarrow)
$$

Hence $\vdash_{E_{-i} F_{-i}} \Delta^{\prime} \rightarrow \Lambda^{\prime}, \neg \bigvee_{s_{i}} D_{i}^{C}\left(s_{i}\right)$ and $\vdash_{E_{-i} F_{-i}} \Delta^{\prime}, d_{i}\left(\mathbf{s}_{i 1}\right) \rightarrow \Lambda^{\prime}$. Looking the latter sequent and applying Theorem 6.1 to this, we have $d_{i}\left(\mathbf{s}_{i 1}\right) \in \Lambda^{\prime}$.

Now, consider the former. Let $P^{\prime}$ be the subproof of the proof of $P$ whose endsequent is $\Delta^{\prime} \rightarrow \Lambda^{\prime}, \neg \bigvee_{s_{i}} D_{i}^{C}\left(s_{i}\right)$. Consider the ancestors of $d_{i}\left(\mathbf{s}_{i 1}\right)$ in $\Lambda^{\prime}$. Since $d_{i}\left(\mathbf{s}_{i 1}\right)$ does not occur in $\Delta^{\prime}$, the uppermost ancestor of $d_{i}\left(\mathbf{s}_{i 1}\right)$ in $\Lambda^{\prime}$ is a thinning formula of (Th) and its descendant is never a side formula of an operational inference or $(\mathrm{B} \rightarrow \mathrm{B})$. Hence, we can delete all occurrences of these ancestors, $d_{i}\left(\mathbf{s}_{i 1}\right)$, from $P^{\prime}$. Hence we have 


$$
\vdash_{E_{-i} F_{-i}} \Delta^{\prime}, \rightarrow \Lambda^{\prime \prime}, \neg \bigvee_{s_{i}} D_{i}^{C}\left(s_{i}\right),
$$

where $\Lambda^{\prime \prime}=\Lambda^{\prime}-\left\{d_{i}\left(\mathbf{s}_{i 1}\right)\right\}$. Since $\Delta^{\prime} \subseteq \Delta=g_{i}^{+} \cup \bigcup_{j \neq i} \mathrm{~B}_{j}\left(\Gamma_{i j}\right)$ and $\Lambda^{\prime \prime} \subseteq \hat{g}_{i}^{-}$, we have, by $(\mathrm{Th})$,

$$
\vdash_{E_{-i} F_{-i}} \hat{g}_{i}^{+}, \bigcup_{j \neq i} \mathrm{~B}_{j}\left(\Gamma_{i j}\right) \rightarrow \hat{g}_{i}^{-}, \neg \bigvee_{s_{i}} D_{i}^{C}\left(s_{i}\right) .
$$

We have $\vdash_{E_{-i} F_{-i}} \hat{g}_{i}, \bigcup_{j \neq i} \mathrm{~B}_{j}\left(\Gamma_{i j}\right) \rightarrow \neg \bigvee_{s_{i}} D_{i}^{C}\left(s_{i}\right)$. This is equivalent to $\vdash_{E_{i} F_{i}}$ $\mathrm{B}_{i}\left(\hat{g}_{i}\right), \bigcup_{j \neq i} \mathrm{~B}_{i} \mathrm{~B}_{j}\left(\Gamma_{i j}\right) \rightarrow \mathrm{B}_{i}\left(\neg \bigvee_{s_{i}} D_{i}^{C}\left(s_{i}\right)\right)$ by Lemmas 6.4 and 6.5.

\section{Concluding remarks}

We have developed a theory of prediction-decision making in game situations. Each player has a prediction-decision criterion involving only shallow interpersonal introspections. We have shown that an outward statement on decision makings for all the players is decomposed into each individual statement, and that an individual statement has an inner parallelism to the component statements. Therefore, discussions in Section 8 can be used cyclically until the bases of criteria are reached. We have considered the behavior of minimal inferential structures in Section 7.

Various examples of prediction-decision criteria are considered together with some games. These examples would suggest more general treatments of minimal inferential structures. Compound criteria of prediction-decision criteria suggested in Section 9 are important examples for further developments, since they depart considerably from the symmetric treatments of the decision criterion for an individual player himself and his prediction on other players. As a whole, we would like to convey the message that our theory enables us to investigate inferential complexities of interpersonal introspections for decision making in game situations.

We emphasized a broad perspective of new research areas where players have shallow epistemic interactions with other people. This includes the possibility that each player has narrow interactions only with relatively few people surrounding himself, which may be read as being suggested in the location game LG. Particularly, our theory may provide candidates for a theory or model derived from individual experiences such as in the inductive game theory of Kaneko and Matsui [5]. Although such areas are in the scope of our theory, we would need more developments of a systematic procedure.

Another remark is on complexities of intrapersonal reasoning in terms of informational content. In principle, it would be possible to have such a consideration. This is a topic to which a lot of attentions have been given recently in the proof-theory literature. Nevertheless, no consensus has been reached, especially, even on purely finite problems. A general development would be difficult also in game theoretical contexts, but some special considerations may be possible since we can often impose special structures on game problems. 
A complexity of intrapersonal reasoning in terms of informational content seems to be related to the number of initial sequents. For example, consider the proof given in (4.1), where game $g^{1}$ is replaced by a general 2-person game $g$ with dominant strategy $\mathbf{s}_{11}$ for player 1 . The proof of $\mathrm{B}_{1}\left[\hat{g}_{1} \rightarrow \operatorname{Dom}_{1}\left(\mathbf{s}_{11}\right)\right]$ has $\left|S_{1}\right| \times$ $\left|S_{2}\right|$ number of initial sequents, which reflects the number of verification for $\mathbf{s}_{11}$ to be a dominant strategy including the comparisons of $\mathbf{s}_{11}$ with itself. In this case, the premise $\hat{g}_{1}$ is treated as stored beliefs and is not counted in the informational content used. If player 1 has the additional information $\bigvee_{s_{1}} \operatorname{Dom}_{1}\left(s_{1}\right)$, then the informational content of $\mathrm{B}_{1}\left[\hat{g}_{1}, \bigvee_{s_{1}} \operatorname{Dom}_{1}\left(s_{1}\right) \rightarrow \operatorname{Dom}_{1}\left(\mathbf{s}_{11}\right)\right]$ has becomes $\left|S_{1}\right|$ under the assumption of no indifferences in $\hat{g}_{1}$. That is, it suffices for player 1 to check his strategies fixing $\mathbf{s}_{11}$. In a future paper, we will investigate into this problem of bounded intrapersonal inferences for decision making.

As pointed out in Section 2, criteria DC1 and DC2 are related to the procedure of iterated elimination of dominated strategies (cf., Moulin [10] and Myerson [11]). The minimal depth of inferential epistemic structure appears to be related to the number of the rounds for the iterated eliminations of dominated strategies. However, the method of iterated eliminations of dominated strategies is not directly a special case of our general definition of a prediction-decision criterion. To consider this method in our approach, the definition of a prediction-decision criterion should be modified.

Finally we give a remark on Aumann and Brandenberger [2], who claimed that common knowledge is not required to have a Nash equilibrium, more specifically, the epistemic depth required to have a Nash equilibrium is at least one. Their claim was stated in a probabilistic model. We may formulate the essential part of their claim in our framework. Consider the statement $(*)$ : if $\vdash \Gamma_{1}, \Gamma_{2} \rightarrow$ $\mathrm{B}_{1}\left(D_{1}\left(s_{1}\right)\right) \wedge \mathrm{B}_{2}\left(D_{2}\left(s_{2}\right)\right)$, then $\vdash \hat{g} \rightarrow \operatorname{Nash}\left(s_{1}, s_{2}\right)$, where $\vdash$ is the provability relation of some epistemic logic, say, $\mathrm{KD}^{n}$. Aumann and Brandenberger's [2] claim is interpreted as follows: we may find some condition on $\Gamma_{1}, \Gamma_{2}$ as well as $D_{1}, D_{2}$ for $(*)$ so that the epistemic depths for these formulae are 1. From our point of view, there are many prediction-decision criteria satisfying this $(*)$, for example, DC1.

The question of whether or not common knowledge is required for the classical (ex ante) Nash equilibrium argument is not to find epistemic requirement for $(*)$, but is to find epistemic requirements of the classical Nash equilibrium argument itself. The latter is discussed in Sections 7 and 8 of Kaneko [4].

\section{Appendix: Treatment of Axiom 4}

In Kaneko [4], multi-modal epistemic logic $\mathrm{KD} 4^{n}$ is treated as central among various candidates. On the other hand, we exclude Axiom 4: $B_{i}(A) \supset B B_{i}(A)$ in our epistemic logic $\mathrm{GL}_{E F}$ of shallow depths, i.e., $\mathrm{GL}_{E F}$ is of KD-type. It is a reason for this exclusion that our focus is the consideration of interpersonal introspections but not intrapersonal ones. Nevertheless, it may be a natural question what would happen if Axiom 4 is included. In this appendix, we argue that the results given in this paper essentially remain to hold even if Axiom 4 is included. 
The relation of $\mathrm{KD}^{n}$ to $\mathrm{KD}^{n}$ is just an addition of Axiom 4 to $\mathrm{KD}^{n}$. On the other hand, to incorporate Axiom 4 to our $\mathrm{GL}_{E F}$ keeping the basic developments such as the cut-elimination theorem as well as completeness result, we need to modify the basic definitions of depths and epistemic structures.

First, we change $N^{<\omega}$ into $N^{<\omega>}=\left\{\left(i_{1}, \ldots, i_{m}\right): i_{1}, \ldots, i_{m} \in N, m \geq 0\right.$ and $i_{t} \neq i_{t+1}$ for $\left.t=1, \ldots, m-1\right\}$. Also, we change the concatenation $\circ$ to $*$ so that for $e=\left(i_{1}, \ldots, i_{m}\right), e^{\prime}=\left(j_{1}, \ldots, j_{k}\right) \in N^{<\omega>}, e * e^{\prime}=\left(i_{1}, \ldots, i_{m}, j_{2}, \ldots, j_{k}\right)$ if $i_{m}=j_{1}$ and $e * e^{\prime}=\left(i_{1}, \ldots, i_{m}, j_{1}, \ldots, j_{k}\right)$ if $i_{m} \neq j_{1}$. Here, the repetitive occurrences of the same $i$ is excluded, since Axiom 4 takes care of such a repetition. Then we change $\delta^{r}$ into $\delta$ so that $\delta$ is defined by conditions D0-D4 with $*$ instead of $\circ$ for D4. For example, $\delta\left(\mathrm{B}_{1} \mathrm{~B}_{1}(p)\right)=\{(1)\}$ but $\delta^{r}\left(\mathrm{~B}_{1} \mathrm{~B}_{1}(p)\right)=\{(1,1)\}$. Descriptive and inferential epistemic structures $E$ and $F$ are now assumed to be subsets of $N^{<\omega\rangle}$ satisfying (3.4). Then the KD4-type $\mathrm{GL}_{E F}^{4}$ is defined by the above list of the axiom and inference rules only with the replacement of the distribution rule by

$$
\frac{\mathrm{B}_{e * i}\left[\Gamma, \mathrm{B}_{i}(\Lambda) \rightarrow \Theta\right]}{\mathrm{B}_{e}\left[\mathrm{~B}_{i}(\Gamma \cup \Lambda) \rightarrow \mathrm{B}_{i}(\Theta)\right]}\left(\mathrm{B}_{i} \rightarrow \mathrm{B}_{i}\right)^{4} \text {, where }|\Theta| \leq 1 \text { and } i \in N
$$

The provability of $\mathrm{GL}_{E F}^{4}$ is denoted by $\vdash_{E F}^{4}$. In the modified logic $\mathrm{GL}_{E F}^{4}$, any formula in $\Gamma \cup \Theta$ in the upper sequent of $\left(\mathrm{B}_{i} \rightarrow \mathrm{B}_{i}\right)^{4}$ is a side formula, and any one in $\mathrm{B}_{i}(\Gamma) \cup \mathrm{B}_{i}(\Theta)$ in the lower sequent is a principal formula.

In $\mathrm{GL}_{E F}^{4}$, Axiom 4 is realized in the following sense:

$$
\frac{\mathrm{B}_{e * i}\left[\mathrm{~B}_{i}(A) \rightarrow \mathrm{B}_{i}(A)\right]}{\mathrm{B}_{e}\left[\mathrm{~B}_{i}(A) \rightarrow \mathrm{B}_{i} \mathrm{~B}_{i}(A)\right]}\left(\mathrm{B}_{i} \rightarrow \mathrm{B}_{i}\right)^{4}
$$

Since we are now using $\delta$, we have no constraints on the repetitions of $\mathrm{B}_{i}$. That is, when $e=\left(i_{1}, \ldots, i_{m}\right)$ and $i_{m}=i$, we have $e * i=e$ itself. To see that $\mathrm{GL}_{E F}^{4}$ captures Axiom 4, we can show the following: Let $\Gamma$ and $\Theta$ be any finite sets of formulae in $\mathscr{P}$ and $E$ an epistemic structure with $\delta(\Gamma \cup \Theta) \subseteq E \subseteq N^{<\omega>}$. Then

$$
\vdash_{\mathrm{KD} 4^{n}} \Gamma \rightarrow \Theta \text { if and only if } \vdash_{E E}^{4} \Gamma \rightarrow \Theta,
$$

where $\vdash_{\mathrm{KD}} 4^{n}$ is the provability of $\mathrm{KD}^{n}$ in the sequent form (cf., Kaneko [4], Section 4.3). This is a variant of Theorem 4.2 for $\mathrm{KD} 4^{n}$.

The cut-elimination theorem (Theorem 4.3) holds for $\mathrm{GL}_{E F}^{4}$, and also, the semantics for $\mathrm{GL}_{E F}^{4}$ has been developed (Kaneko and Suzuki [7] and [8]). If $P^{\prime}$ is a proof of $\mathrm{B}_{e}[\Gamma \rightarrow \Theta]$, then the cut-elimination theorem gives a cut-free proof $P$ of the same endsequent $\mathrm{B}_{e}[\Gamma \rightarrow \Theta]$. We emphasize that a cut-free proof $P$ enjoys the subformula property that any formula in $P$ occurs as a subformula also in the endsequent $\mathrm{B}_{e}[\Gamma \rightarrow \Theta]$ of $P$.

The changes from $\mathrm{GL}_{E F}$ to $\mathrm{GL}_{E F}^{4}$ may look small. However, $\mathrm{GL}_{E F}^{4}$ is more difficult to be handled than $\mathrm{GL}_{E F}$ in considerations of meta-theoretical arguments. In $\mathrm{GL}_{E F}^{4}$, for example, Theorem 6.3, Lemmas 6.4 and 6.5 need some additional assumptions and their proofs become much more complicated. Nevertheless, as far as formulae are restricted to ones compatible with our restriction to purely 
interpersonal introspections, we can prove that provability $\vdash_{E F}^{4}$ is equivalent to $\vdash_{E F}$.

Theorem 11.1. Let $E$ and $F$ be epistemic structures with $F \subseteq E$ which are subsets of $N^{<\omega>}$, and let $\delta^{r}(\Gamma \cup \Lambda) \subseteq N^{<\omega>}$. Then $\vdash_{E F}^{4} \Gamma \rightarrow \Lambda$ if and only if $\vdash_{E F} \Gamma \rightarrow \Lambda$.

The if part is automatically implied, but the only-if part needs a long proof, which will be given below.

Proof of Theorem 11.1. Suppose $\vdash_{E F}^{4} \Gamma \rightarrow \Lambda$. There is a cut-free proof $P$ of $\Gamma \rightarrow \Lambda$ in logic $\mathrm{GL}_{E F}^{4}$. Note that since $P$ is cut-free, it satisfies the subformula property that any formula in $P$ occurs as a subformula in the endsequent $\Gamma \rightarrow \Lambda$ of $P$.

Then $P$ may contain some application of $\left(\mathrm{B}_{i} \rightarrow \mathrm{B}_{i}\right)^{4}$ of the following two types:

$$
\begin{gathered}
\text { (A): } \frac{\mathrm{B}_{e}\left[\Delta_{1}, \mathrm{~B}_{i}\left(\Delta_{2}\right) \rightarrow \Theta_{1}\right]}{\mathrm{B}_{e}\left[\mathrm{~B}_{i}\left(\Delta_{1} \cup \Delta_{2}\right) \rightarrow \mathrm{B}_{i}\left(\Theta_{1}\right)\right]}\left(\mathrm{B}_{i} \rightarrow \mathrm{B}_{i}\right)^{4}, \\
(\mathrm{~B}): \frac{\mathrm{B}_{\left(i_{1}, \ldots, i_{m}\right)}\left[\Delta_{1}, \mathrm{~B}_{i}\left(\Delta_{2}\right) \rightarrow \Theta_{1}\right]}{\mathrm{B}_{\left(i_{1}, \ldots, i_{m-1}\right)}\left[\mathrm{B}_{i}\left(\Delta_{1} \cup \Delta_{2}\right) \rightarrow \mathrm{B}_{i}\left(\Theta_{1}\right)\right]}\left(\mathrm{B}_{i} \rightarrow \mathrm{B}_{i}\right)^{4},
\end{gathered}
$$

where $e=\left(i_{1}, \ldots, i_{m}\right)$ and $i=i_{m}$. It suffices to find another proof $P^{*}$ of $\Gamma \rightarrow \Lambda$ where there are no applications of $\left(\mathrm{B}_{i} \rightarrow \mathrm{B}_{i}\right)^{4}$ of type (A) and every applications of $\left(\mathrm{B}_{i} \rightarrow \mathrm{B}_{i}\right)^{4}$ of type (B) has "empty" $\mathrm{B}_{i}\left(\Delta_{2}\right)$. Specifically, we will modify $P$ into $P^{*}$ so that we "delete" the type (A)'s and change the type (B)'s into

$$
\frac{\mathrm{B}_{\left(i_{1}, \ldots, i_{m}\right)}\left[\Delta_{1}, \Delta_{2} \rightarrow \Theta_{1}\right]}{\mathrm{B}_{\left(i_{1}, \ldots, i_{m-1}\right)}\left[\mathrm{B}_{i}\left(\Delta_{1} \cup \Delta_{2}\right) \rightarrow \mathrm{B}_{i}\left(\Theta_{1}\right)\right]}\left(\mathrm{B}_{i} \rightarrow \mathrm{B}_{i}\right) .
$$

Then we will show that $P^{*}$ is a proof of $\Gamma \rightarrow \Lambda$ in logic GL $\mathrm{GF}_{E F}$.

Since $\delta^{r}(\Gamma \cup \Lambda) \subseteq N^{<\omega>}$, we have

$$
\delta^{r}(A) \subseteq N^{<\omega>} \text { for any formula } A \text { occurring in } P .
$$

Indeed, consider a formula $A$ in any sequent $\mathrm{B}_{e}[\Delta \rightarrow \Lambda]$ in $P$. By the subformula property of $P, A$ occurs as a subformula in the endsequent $\Gamma \rightarrow \Theta$. Hence $\delta^{r}(A) \subseteq N^{<\omega>}$.

Now, consider a particular application $\eta$, in $P$, of an inference of the form:

$$
\eta: \frac{\mathrm{B}_{\left(i_{1}, \ldots, i_{m}\right)}\left[\Delta_{1}, \mathrm{~B}_{i}\left(\Delta_{2}\right) \rightarrow \Theta_{1}\right]}{\mathrm{B}_{\left(i_{1}, \ldots, i_{m-1}\right)}\left[\mathrm{B}_{i}\left(\Delta_{1} \cup \Delta_{2}\right) \rightarrow \mathrm{B}_{i}\left(\Theta_{1}\right)\right]}\left(\mathrm{B}_{i} \rightarrow \mathrm{B}_{i}\right)^{4} .
$$

where $e=\left(i_{1}, \ldots, i_{m}\right)$ and $i_{m}=i$. Let $Q$ be the part of $P$ consisting of the ancestor sequents, with the same outer $\mathrm{B}_{e}[\cdots]$, of the upper sequent of $\eta$. We stipulate that $Q$ includes the upper sequent of $\eta$. The uppermost sequent of $Q$ is either (a): an initial sequent $\mathrm{B}_{e}[D \rightarrow D]$ or $(\mathrm{b})$ : the lower sequent of $\left(\mathrm{B}_{i_{m+1}} \rightarrow \mathrm{B}_{i_{m+1}}\right)^{4}$ with $i_{m+1} \neq i$.

First, we list several facts on $P$, and using these facts, $P$ will be modified into $P^{*}$. 
(1): First, the succedent $\Xi$ of $\mathrm{B}_{e}[\Pi \rightarrow \Xi]$ in $Q$ does not have a formula of the form $\mathrm{B}_{i}(A)=\mathrm{B}_{i_{m}}(A)$. Indeed, if $\Xi$ has a formula $\mathrm{B}_{i}(A)$, then its descendant of occurs as a subformula in $\Delta_{1} \cup \Delta_{2} \cup \Theta_{1}$ of the lower sequent of $\eta$, which implies we have $\left(i, i, j_{1}, \ldots, j_{\ell}\right) \in \delta^{r}\left(\mathrm{~B}_{i}\left(\Delta_{1} \cup \Delta_{2}\right) \cup \mathrm{B}_{i}\left(\Theta_{1}\right)\right)$ for some $\left(j_{1}, \ldots, j_{\ell}\right)$, a contradiction to (11.2).

(2): Consider an initial sequent $\mathrm{B}_{e}[D \rightarrow D]$ in $Q$. Then $D$ cannot be the form $\mathrm{B}_{i}(A)$ by (1).

(3): Consider any application $\eta^{\prime}$ of $\left(\mathrm{B}_{i} \rightarrow \mathrm{B}_{i}\right)^{4}$, in $Q$, of the form:

$$
\eta^{\prime}: \frac{\mathrm{B}_{e}\left[\Lambda_{1}, \mathrm{~B}_{i}\left(\Lambda_{2}\right) \rightarrow \Xi_{1}\right]}{\mathrm{B}_{e}\left[\mathrm{~B}_{i}\left(\Lambda_{1} \cup \Lambda_{2}\right) \rightarrow \mathrm{B}_{i}\left(\Xi_{1}\right)\right]}\left(\mathrm{B}_{i} \rightarrow \mathrm{B}_{i}\right)^{4} .
$$

By (1), $\Xi_{1}=\emptyset$.

Consider an arbitrary occurrence $\xi$ of a formula $\mathrm{B}_{i}(A)=\mathrm{B}_{i_{m}}(A)$ in $Q$.

(4): First, $\xi$ is not a side formula of any inference. Suppose, on the contrary, that it is a side formula of some inference rule $I$. Let $I$ be $\left(\mathrm{B}_{i} \rightarrow \mathrm{B}_{i}\right)$ such as $\eta^{\prime}$ of (2). Then $\mathrm{B}_{i}(A)$ is in $\Lambda_{1} \cup \Xi_{1}$, and we have $\mathrm{B}_{i}(A) \in \Lambda_{1}$ by $\Xi_{1}=\emptyset$ by (3), which implies that $\mathrm{B}_{i} \mathrm{~B}_{i}(A) \in \mathrm{B}_{i}\left(\Lambda_{1}\right)$, a contradiction to (11.2). Note that it may be the case that $\xi$ is in $\mathrm{B}_{i}\left(\Lambda_{2}\right)$ in the upper sequent.

For any operational inference $I$, we can show in the same manner that $\xi$ is not a side formula of $I$.

(5): The uppermost ancestor of $\xi$, having the form $\mathrm{B}_{i}(A)$, in $Q$ is not a principal formula of $\left(\mathrm{B}_{i_{m+1}} \rightarrow \mathrm{B}_{i_{m+1}}\right)^{4}$, since $i_{m+1} \neq i_{m}$.

(6): By (1), (2), (3),(4) and (5), the uppermost ancestor, having the form $\mathrm{B}_{i}(A)$, of $\xi$ in $Q$ is either an thinning formula of $(\mathrm{Th})$ or a principal formula of $\left(\mathrm{B}_{i} \rightarrow\right.$ $\mathrm{B}_{i}$ ) of the type $\mathrm{B}$, and every descendant of $\xi$ occurs as $\mathrm{B}_{i}(A)$ in $Q$.

Now, we replace all occurrences of any formula of the form of $\mathrm{B}_{i}(A)=$ $\mathrm{B}_{i_{m}}(A)$ in $Q$ by $A$. The new part is denoted by $Q^{\prime}$. Let $P^{\prime}$ be the tree obtained by replacing $Q$ by $Q^{\prime}$. Now we show that the part $Q^{\prime}$ is correctly constructed with the inference rules for $\mathrm{GL}_{E F}$. Since $Q^{\prime}$ is affected for a sequent including a formula of the form $\mathrm{B}_{i}(A)$. By (4), we need only to consider an application $\eta^{\prime}$ of $\left(\mathrm{B}_{i} \rightarrow \mathrm{B}_{i}\right)^{4}$ in (3): $\eta^{\prime}$ is changed into

$$
\frac{\mathrm{B}_{e}\left[\Lambda_{1}, \Lambda_{2} \rightarrow\right]}{\mathrm{B}_{e}\left[\Lambda_{1} \cup \Lambda_{2} \rightarrow\right]}
$$

Thus the upper and lower sequents are the same. This is regarded as (Th). Hence $Q^{\prime}$ is correctly connected in $\mathrm{GL}_{E F}$. The lowermost sequent of $Q^{\prime}$ is:

$$
\mathrm{B}_{\left(i_{1}, \ldots, i_{m}\right)}\left[\Delta_{1} \cup \Delta_{2} \rightarrow \Theta\right] .
$$

Hence we have

$$
\frac{\mathrm{B}_{\left(i_{1}, \ldots, i_{m}\right)}\left[\Delta_{1}, \Delta_{2} \rightarrow \Theta\right]}{\mathrm{B}_{\left(i_{1}, \ldots, i_{m-1}\right)}\left[\mathrm{B}_{i}\left(\Delta_{1} \cup \Delta_{2}\right) \rightarrow \mathrm{B}_{i}(\Theta)\right]}\left(\mathrm{B}_{i} \rightarrow \mathrm{B}_{i}\right) .
$$


In this manner, we change the part of each application of the form $\eta$ in the above way. The resulting $P^{*}$ is a proof of $\Gamma \rightarrow \Lambda$ in $\mathrm{GL}_{E F}$.

\section{List of Some Symbols:}

$N=\{1, \ldots, n\}:$ the set of players;

$S_{i}=\left\{\mathbf{s}_{i 1}, \ldots, \mathbf{s}_{i \ell_{i}}\right\}:$ the set of strategies of player $i$;

$g=\left(g_{1}, \ldots, g_{n}\right):$ a game consisting of payoff functions $g_{1}, \ldots, g_{n}$;

$N^{<\omega}=\left\{\left(i_{1}, \ldots, i_{m}\right): i_{1}, \ldots, i_{m} \in N\right.$ and $\left.m \geq 0\right\}$

$N^{<\omega>}=\left\{\left(i_{1}, \ldots, i_{m}\right) \in N^{<\omega}: i_{t} \neq i_{t+1}\right.$ for $\left.t=1, \ldots, m-1\right\}$;

$\epsilon:$ the null sequence;

$\neg, \supset, \bigwedge, \bigvee:$ logical connectives;

$\mathscr{P}$ : the set of formulae;

$A, B, C, D$; formulae;

$\mathrm{B}_{i}(A)$ : player $i$ believes $A$;

$\hat{g}_{i}$ : the set of preferences expressing payoff function $g_{i}$;

$\top: \neg p \vee p ;$ and $\perp: \neg p \wedge p$;

$\delta^{r}(A)$ : the epistemic depths of formula $A$;

$E$ and $F$ : epistemic structures with $F \subseteq E$;

$E_{i}=\left\{\left(i_{1}, \ldots, i_{m}\right) \in E: i_{1}=i\right\} \cup\{\epsilon\} ;$ and $E_{-i}=\left\{\left(i_{2}, \ldots, i_{m}\right):\left(i_{1}, \ldots, i_{m}\right) \in E\right\}$;

$\mathscr{P}_{E}=\left\{A \in \mathscr{P}: \delta^{r}(A) \subseteq E\right\}$

$\Gamma, \Theta, \Delta$ : finite sets of formulae;

$\Phi$ : a nonempty finite set of formulae;

$\mathrm{B}_{i}(\Phi):=\left\{\mathrm{B}_{i}(A): A \in \Phi\right\}$

$\mathrm{B}_{e}[\Gamma \rightarrow \Theta]$ : a thought sequent;

$\mathrm{GL}_{E F}$ : epistemic logic of shallow depths;

$\vdash_{E F}$ : the provability relation of $\mathrm{GL}_{E F}$;

$\operatorname{Best}_{i}\left(s_{i} \mid s_{-i}\right): s_{i}$ is a best response to $s_{-i}$;

$\operatorname{Dom}_{i}\left(s_{i}\right): s_{i}$ is a dominant strategy;

$\operatorname{Und}_{i}\left(s_{i}\right): s_{i}$ is an undominated strategy;

$\mathscr{D}_{i}=\left\{D_{i}\left(s_{i}\right): s_{i} \in S_{i}\right\}:$ a prediction-decision criterion;

$\mathscr{T}_{i}=\left\{\operatorname{Pre}_{i j}\left(s_{j}\right): s_{j} \in S_{j}\right.$ and $\left.j \in N-\{i\}\right\}:$ prediction criterion of player $i$;

$\mathscr{R}_{i}=\left\{\operatorname{Res}_{i}\left(s_{i} \mid s_{-i}\right): s_{i} \in S_{i}\right.$ and $\left.s_{-i} \in S_{-i}\right\}:$ valuation criterion of player $i$;

$P_{i j}^{0}\left(s_{j}\right)=\top$.

\section{References}

1. Aumann, R. J., Maschler, M.: Some thoughts on the minimax principle. Management Sciences 18, 54-63 (1972)

2. Aumann, R. J., Brandenberger, A.: Epistemic conditions for Nash equilibrium. Econometrica 63, 1161-1180 (1995)

3. Gentzen, G.: Untersuchungen über das logische Schliessen. Mathematische Zeitschrift 39, 176210, 405-431 (1935). English translation: Investigations into logical deduction. In: Szabo, M. E. (ed.) The Collected Papers of Gerhard Gentzen. Amsterdam: North-Holland 1969

4. Kaneko, M.: Introduction to epistemic logics and their game theoretical applications. Economic Theory (this issue) 
5. Kaneko, M., Matsui, A.: Inductive game theory: discrimination and prejudices. Journal of Public Economic Theory 1, 101-137 (1999). Errata: 3, 347 (2001)

6. Kaneko, M., Nagashima, T.: Game logic and its applications II. Studia Logica 58, 273-303 (1977)

7. Kaneko, M., Suzuki, N.-Y.: Semantics of epistemic logics of shallow depths for game theory. IPPS, DP. No. 814, University of Tsukuba (1999)

8. Kaneko, M., Suzuki, N.-Y.: Epistemic models of shallow depths and decision making in games. IPPS, DP. No. 828, University of Tsukuba (1999)

9. Kaneko, M., Suzuki, N.-Y.: Epistemic logics of shallow depths and game theoretical applications. In: Wolter, F. et al. (eds.) Advances in modal logics, Vol.3. Center for the study of language and information Publisher (2001) (to appear)

10. Moulin, H.: Game theory for the social sciences. New York: New York University Press 1982

11. Myerson, R.: Game theory: analysis of conflict. Cambridge: Harvard University Press 1991

12. von Neumann, J.: Zur Theorie der Gesellschaftsspiele. Mathematische Annalen 100, 295-320 (1928)

13. von Neumann, J.: Über ein ökonomisches Gleichungssystem und eine Verallgemeinerung des Brouwerschen Fixpunktsatzes. Ergebnisse eines Mathematischen Kolloquiums 8, 73-83 (1937). English translation: A Model of general economic equilibrium. Review of Economic Studies 13, $1-9(1945)$ 